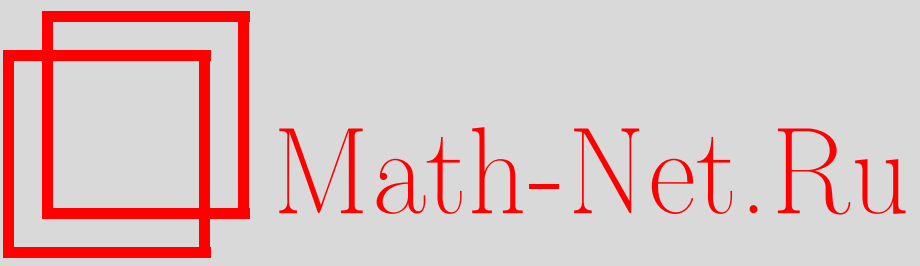

Ю. С. Нефедова, И. Г. Шевцова, О неравномерных оценках скорости сходимости в центральной предельной теореме, Теория вероятн. и ее примен., 2012, том 57, выпуск 1, 62-97

DOI: https://doi.org/10.4213/tvp4432

Использование Общероссийского математического портала Math-Net.Ru подразумевает, что вы прочитали и согласны с пользовательским соглашением

http: //www . mathnet.ru/rus/agreement

Параметры загрузки :

IP : 34.239 .49 .27

26 апреля 2023 г., 09:48:32 
ТЕОРИЯ ВЕРОЯТНОСТЕЙ

T O M 57

И ЕЕ ПРИМЕНЕНИЯ

Выпуск 1

2012

(C) 2012 г. $\quad$ НЕФЕДОВА Ю. С.* , ШЕВЦОВА И. Г.*

\title{
О НЕРАВНОМЕРНЫХ ОЦЕНКАХ СКОРОСТИ СХОДИМОСТИ В ЦЕНТРАЛЬНОЙ ПРЕДЕЛЬНОЙ ТЕОРЕМЕ ${ }^{1)}$
}

\begin{abstract}
Уточнены верхние оценки абсолютной постоянной в неравномерных оценках скорости сходимости в центральной предельной теореме для сумм независимых одинаково распределенных случайных величин, обладающих моментами порядка $2+\delta, 0<\delta \leqslant 1$. В частности, показано, что при существовании третьего момента эта константа не превосходит 18.2. Также показано, что абсолютную константу в указанных оценках можно заменить функцией $C^{*}(|x|, \delta)$ от аргумента $x$ изучаемой разности допредельной и предельной нормальной функций распределения, для которой найдена положительная ограниченная невозрастающая мажоранта, причем для $\delta=1$ эта мажоранта является асимптотически точной (неулучшаемой) при $x \rightarrow \infty$ и уточняет оценки В. Никулина [48] при всех $x$. Для случая же $\delta \in(0,1)$ подобный результат приводится впервые. Как следствие получены верхние оценки функций Колмогорова, являющихся аналогами правильных и асимптотически правильных констант в (равномерном) неравенстве Берри-Эссеена.
\end{abstract}

Ключевые слова и фразы: центральная предельная теорема, неравномерная оценка скорости сходимости, неравенство БерриЭссеена, абсолютная постоянная, асимптотически правильная постоянная.

1. Введение и формулировка основного результата. Пусть $X_{1}, X_{2}, \ldots$ - последовательность независимых случайных величин с общей функцией распределения $F$ и таких, что $\mathbf{E} X_{1}=0, \mathbf{E} X_{1}^{2}=1$, $\mathbf{E}\left|X_{1}\right|^{2+\delta}=\beta_{2+\delta}<\infty$ для некоторого $\delta \in(0,1]$. Обозначим $\mathscr{F}_{2+\delta}$ множество всех функций распределения $F$ случайной величины $X_{1}$, удовлетворяющих указанным условиям. Пусть $F_{n}(x)=\mathbf{P}\left(X_{1}+\cdots+X_{n}<x\right)=$

*Московский государственный университет им. М. В. Ломоносова, факультет вычислительной математики и кибернетики, Ленинские горы, 119991 Москва, Россия; e-mail: julia_n@inbox.ru, ishevtsova@cs.msu.su

1) Работа выполнена при поддержке Российского фонда фундаментальных исследований (проекты 11-01-00515a, 11-07-00112a), а также Министерством образования и науки РФ (грант МК-581.2010.1, государственные контракты П1181, П958, П779 и 16.740.11.0133 в рамках ФЦП «Научные и научно-педагогические кадры инновационной России на 2009-2013 годы»). 
$F^{* n}(x), \varphi(x)$ и $\Phi(x)$ - соответственно плотность и функция распределения стандартного нормального закона. Положим

$$
\Delta_{n}(x)=\left|F_{n}(x \sqrt{n})-\Phi(x)\right|, \quad x \in \mathbf{R}, \quad n \geqslant 1 .
$$

Известно, что при указанных условиях существует (см., например, [22]) абсолютная положительная конечная константа $C_{0}(\delta)$ такая, что

$$
\sup _{x} \Delta_{n}(x) \leqslant C_{0}(\delta) L_{n}^{2+\delta}, \quad \text { где } L_{n}^{2+\delta}=\frac{\beta_{2+\delta}}{n^{\delta / 2}} .
$$

Данное неравенство впервые было доказано в 1941-1942 гг. при $\delta=1$ независимо Э. Берри [31] и К.-Г. Эссееном [34]. При $0<\delta<1$ его можно вывести из полученной в 1945 г. Эссееном [36] оценки

$$
\sup _{x} \Delta_{n}(x) \leqslant A_{1}(\delta)\left(L_{n}^{2+\delta}+\left(L_{n}^{2+\delta}\right)^{1 / \delta}\right)
$$

справедливой для необязательно одинаково распределенных слагаемых, где $A_{1}(\delta)$ зависит только от $\delta$. Неравенство (1) также можно вывести из оценки

$$
\sup _{x} \Delta_{n}(x) \leqslant A_{2} \cdot \mathbf{E} X_{1}^{2} g\left(X_{1}\right) / g(\sqrt{n}),
$$

доказанной в 1963 г. М. Кацем [39] для одинаково распределенных слагаемых, и обобщенной позже на случай разнораспределенных слагаемых В. В. Петровым [21], где $g(x)$ - четная функция, не убывающая вместе с $x / g(x), A_{2}$ - положительная конечная абсолютная постоянная. Недавно В. Ю. Королев и С. В. Попов [41] показали, что в общем случае необязательно одинаково распределенных слагаемых $A_{2} \leqslant 2.011$, какой бы ни была функция $g$, удовлетворяющая указанным выше условиям.

Уточнению оценок констант $C_{0}(\delta)$ при разных $\delta$ посвящено много работ (см. исторические обзоры в [11], [12], [40], [29]). В последнее время усилиями В. Ю. Королева, И. Г. Шевцовой и И.С. Тюрина верхнюю оценку для $C_{0}(1)$ удалось существенно снизить. В работе Тюрина $[26]$ доказана оценка $C_{0}(1) \leqslant 0.4785$. Королевым и Шевцовой получена оценка $C_{0}(1) \leqslant 0.4784$ как следствие доказанного в работах [12], [40] неравенства

$$
\sup _{x} \Delta_{n}(x) \leqslant 0.33477\left(\beta_{3}+0.429\right) / \sqrt{n},
$$

являющегося структурным уточнением неравенства (1) (так как $0.33477\left(\beta_{3}+0.429\right) \leqslant 0.33477(1+0.429) \beta_{3}<0.4784 \beta_{3}$ в силу условия $\left.\beta_{3} \geqslant 1\right)$. Нижняя оценка $C_{0}(1)$ приведена в статье Эссеена [35]. Таким образом, относительно $C_{0}(1)$ в настоящий момент известно [35], [12], [40], 
чTо

$$
0.4097 \approx \frac{\sqrt{10}+3}{6 \sqrt{2 \pi}} \leqslant C_{0}(1)<0.4784 .^{2)}
$$

Наилучшие на сегодняшний день верхние оценки констант $C_{0}(\delta)$ при $0<\delta<1$ получены в работе Григорьевой и Шевцовой [5], нижние оценки доказаны Шевцовой в [29] (см. табл. 1).

Таблица 1. Двусторонние оценки констант $C_{0}(\delta)$ для некоторых $\delta \in$ $(0,1)$, полученные в работах [5], [29].

\begin{tabular}{||c|c|c||c|c|c||c|c|c||}
\hline$\delta=$ & $C_{0}(\delta) \geqslant$ & $C_{0}(\delta) \leqslant$ & $\delta=$ & $C_{0}(\delta) \geqslant$ & $C_{0}(\delta) \leqslant$ & $\delta=$ & $C_{0}(\delta) \geqslant$ & $C_{0}(\delta) \leqslant$ \\
\hline 0.9 & 0.2383 & 0.5383 & 0.6 & 0.2651 & 0.6276 & 0.3 & 0.3257 & 0.6195 \\
0.8 & 0.2446 & 0.5723 & 0.5 & 0.2803 & 0.6413 & 0.2 & 0.3603 & 0.6094 \\
0.7 & 0.2534 & 0.6026 & 0.4 & 0.3000 & 0.6342 & 0.1 & 0.4097 & 0.6028 \\
\hline
\end{tabular}

В работах [12], [40] была получена оценка, также являющаяся структурным уточнением неравенства (1)

$$
\sup _{x} \Delta_{n}(x) \leqslant 0.3041\left(\beta_{3}+1\right) / \sqrt{n} \text {. }
$$

Несложно убедиться, что оценка (2) точнее (1) при $\beta_{3} \geqslant 1.93$.

Для случая $\delta=0$ известна тривиальная оценка $\sup _{x} \Delta_{n}(x) \leqslant$ 0.5409 ... [3] (см. лемму 4 ниже). Для этого случая в 1966 г. Л. В. Осипов [18] доказал, что существует такая конечная положительная абсолютная постоянная $A_{3}$, что

$$
\sup _{x} \Delta_{n}(x) \leqslant A_{3} \cdot \mathbf{E} X_{1}^{2} \min \left\{1, \frac{\left|X_{1}\right|}{\sqrt{n}}\right\},
$$

(также см. [23, глава V, §3, теорема 7]). Результат Осипова был доказан для необязательно одинаково распределенных слагаемых. Также не предполагая одинаковой распределенности слагаемых, Л. Падитц показал [54], [56], что для константы $A_{3}$ в (3) справедлива оценка $A_{3} \leqslant 4.77$. В 1986 г. в работе [57] он же отметил, что с учетом леммы 12.2 из монографии [3] с помощью техники, использованной в работах [54], [56], верхнюю оценку константы $A_{3}$ можно снизить до $A_{3} \leqslant 3.51$. По-видимому, не будучи знакомыми с упомянутыми работами Падитца, в 2001 г. Чен и Шао

2) Пока данная статья готовилась к печати, этот результат был улучшен в работе Shevtsova I. On the absolute constants in the Berry-Esseen type inequalities for identically distributed summands, arXiv:1111.6554, 28 November 2011, где доказано неравенство $\sup _{x} \Delta_{n}(x) \leqslant 0.33554\left(\beta_{3}+0.415\right) / \sqrt{n}$, следствием которого является наилучшая на сегодняшний день оценка $C_{0}(1) \leqslant 0.4748$. Независимо в работе Тюрина И. С. «Уточнение остаточного члена в теореме Ляпунова» (см. Теория вероятн. и ее примен., 2011 , т. 56 , в. 4 , с. $808-811)$ получена оценка $C_{0}(1) \leqslant 0.4774$. 
опубликовали работу [32], в которой с помощью метода ТихомироваСтейна неравенство (3) было доказано с константой $A_{3}=4.1$. В недавней работе [9] показано, что $A_{3} \leqslant 2.011$, откуда вытекает равномерная по $\delta \in[0,1]$ оценка $C_{0}(\delta) \leqslant 2.011$.

Оценка скорости сходимости $F_{n}(x \sqrt{n})$ к $\Phi(x)$, устанавливаемая неравенством Берри-Эссеена (1), равномерна по $x$. Но поскольку и $F_{n}(x \sqrt{n})$, и $\Phi(x)$ - функции распределения, должно выполняться соотношение $\Delta_{n}(x) \rightarrow 0$ при $|x| \rightarrow \infty$. Это обстоятельство не учитывается в равномерных оценках. Вместе с тем точность нормальной аппроксимации для функции распределения сумм случайных величин именно при больших значениях аргумента представляет особый интерес, например, при вычислении рисков критически больших потерь. В данной статье мы рассмотрим неравномерные оценки скорости сходимости в центральной предельной теореме.

Вопрос о зависимости остаточного члена в центральной предельной теореме от $n$ и $x$ рассматривался еще в работе Крамера [33] для распределений с экспоненциально убывающими хвостами, т.е. таких, что $\mathbf{E} \exp \left\{a\left|X_{1}\right|\right\}<\infty$ для некоторого $a>0$. Для распределений же, удовлетворяющих рассматриваемым моментным условиям, повидимому, исторически первая оценка величины $\Delta_{n}(x)$ была получена К.-Г.Эссееном [36] в 1945 г. для случая $\delta=1$ и одинаково распределенных слагаемых и имела вид

$$
\Delta_{n}(x) \leqslant \frac{A_{4}\left(\beta_{3}\right)}{\sqrt{n}} \cdot \frac{\ln (2+|x|)}{1+|x|^{3}},
$$

где $A_{4}\left(\beta_{3}\right)$ зависит только от $\beta_{3}$. В работе Л. Д. Мешалкина и Б. А. Рогозина [13] с помощью неравенства сглаживания, отличного от неравенства сглаживания, использованного Эссееном, было доказано существование абсолютной постоянной $A_{5}$ такой, что при всех $x \in \mathbf{R}$ и $n \geqslant 1$

$$
\Delta_{n}(x) \leqslant A_{5} \frac{\beta_{3}}{\sqrt{n}} \cdot \frac{\max \{\ln n, \ln (2+|x|)\}}{1+|x|^{3}},
$$

а также существование абсолютной постоянной $A_{6}$ такой, что при всех $n \geqslant 1$

$$
\sup _{x \in \mathbf{R}}\left(1+x^{2}\right) \Delta_{n}(x) \leqslant A_{6} \cdot L_{n}^{3} .
$$

Результаты работ [36] и [13] затем были усилены и обобщены в работах С. В.Нагаева [15] (для случая одинаково распределенных слагаемых и $\delta=1$ ) и А. Бикялиса [1] (для случая необязательно одинаково распределенных слагаемых и $0<\delta \leqslant 1)$, где было показано, что существуют такие положительные конечные числа $C(\delta)$, что

$$
\sup _{x}\left(1+|x|^{2+\delta}\right) \Delta_{n}(x) \leqslant C(\delta) L_{n}^{2+\delta} .
$$


Вопрос о «правильности» (точности) устанавливаемого оценкой (4) порядка по $n$ и $x$ изучался в работах Л. В. Осипова и В.В. Петрова [19], А. Бикялиса [2], К. Хейди [38], Т. Накаты [45], Р. Михеля [42], В. В. Петрова [24], Л. В. Розовского [25].

Впервые верхние оценки для $C(\delta)$ были получены в работах Л. Падитца [50], [51], [52], [53] для необязательно одинаково распределенных слагаемых. В частности, в своей первой работе на эту тему [52], опубликованной лишь в 1978 г., для $C(1)$ им была получена оценка, превосходящая 1955. Затем в [53] приведены оценки

$$
\begin{gathered}
C(0.9) \leqslant 820.4, \quad C(0.7) \leqslant 569.5, \quad C(0.5) \leqslant 376.7, \\
C(0.3) \leqslant 241.4, \quad C(0.1) \leqslant 151.3 .
\end{gathered}
$$

В [51] было показано, что $C(1) \leqslant 114.7$. В работе Р. Михеля [43] для случая одинаково распределенных слагаемых было показано, что $C(1) \leqslant$ $C_{0}(1)+8(1+e)$, что с учетом оценки для $C_{0}(1)$, полученной в работе [12], влечет оценку $C(1) \leqslant 30.2247$. В работе В. Тысиака [61] для случая $0<\delta \leqslant 1$ и необязательно одинаково распределенных слагаемых были получены оценки

$$
\begin{aligned}
& C(1.0) \leqslant 32.88, \quad C(0.9) \leqslant 29.83, \quad C(0.8) \leqslant 27.21, \quad C(0.7) \leqslant 25.06, \\
& C(0.6) \leqslant 23.41, \quad C(0.5) \leqslant 21.94, \quad C(0.4) \leqslant 20.58, \quad C(0.3) \leqslant 19.32, \\
& C(0.2) \leqslant 18.17, \quad C(0.1) \leqslant 17.05 \text {. }
\end{aligned}
$$

Ш. А. Мирахмедов [14] утверждал, что оценка Михеля $C(1) \leqslant C_{0}(1)+$ $8(1+e)$ справедлива и в общем случае произвольно распределенных слагаемых. Однако вычисления в работах [61], [14] содержали неточности (см. замечания в [20] и [58]). В [20] Л. Падитц и Ш. Мирахмедов получили оценку $C(1) \leqslant 32.153$. В 1986 г. Л. Падитц [57] показал, что $C(1) \leqslant 31.935$. Недавно указанные оценки были уточнены для случая одинаково распределенных слагаемых в работе авторов [16], где было показано, что

$$
\begin{array}{llll}
C(1.0) \leqslant 25.80, & C(0.9) \leqslant 24.23, & C(0.8) \leqslant 22.41, & C(0.7) \leqslant 20.68, \\
C(0.6) \leqslant 19.01, & C(0.5) \leqslant 17.37, & C(0.4) \leqslant 15.69, & C(0.3) \leqslant 14.08, \\
& C(0.2) \leqslant 12.65, & C(0.1) \leqslant 11.37 . &
\end{array}
$$

В статье [4] для случая $\delta=1$ была получена неравномерная оценка уточненной структуры вида

$$
\left(1+|x|^{3}\right) \Delta_{n}(x) \leqslant 22.7707 \cdot\left(\beta_{3}+1\right) / \sqrt{n}, \quad x \in \mathbf{R}, \quad n \geqslant 1, \quad F \in \mathscr{F}_{3} .
$$

При этом, поскольку всегда $\beta_{3} \geqslant 1$, при больших значениях $\beta_{3}$ оценка (5) точнее, чем (4) за счет меньшего значения абсолютной константы. 
В 2001 г. Чен и Шао [32] получили неравномерное уточнение неравенства Осипова (3), построив неравномерную оценку скорости сходимости в центральной предельной теореме, не требующую существования моментов порядка выше двух у суммируемых случайных величин. А именно, если $X_{1}, \ldots, X_{n}$ - независимые случайные величины с $\mathbf{E} X_{i}=0$ и $\mathbf{E} X_{1}^{2}+\cdots+\mathbf{E} X_{n}^{2}=1$, то, как показано в [32], существует константа $C_{C S}$ такая, что для всех $x \in \mathbf{R}$

$$
\Delta_{n}(x) \leqslant C_{C S} \sum_{i=1}^{n}\left\{\frac{\mathbf{E} X_{i}^{2} I\left(\left|X_{i}\right| \geqslant 1+|x|\right)}{(1+|x|)^{2}}+\frac{\mathbf{E}\left|X_{i}\right|^{3} I\left(\left|X_{i}\right|<1+|x|\right)}{(1+|x|)^{3}}\right\} .
$$

Верхним оценкам константы $C_{C S}$ посвящены работы [46], [60], [47]. Примечательно, что эти оценки зависят от значения $x$. В частности, в [47] приведена оценка $C_{C S} \leqslant 76.17$, а также показано, что $C_{C S} \leqslant 39.39$, если $|x| \geqslant 14$.

В работе В. Н. Никулина [17] (также см. [49]) было показано, что для случая $\delta=1$ абсолютную константу $C(1)$ в неравенстве

$$
\frac{\sqrt{n}}{\beta_{3}} \sup _{x \in \mathbf{R}}\left(1+|x|^{3}\right) \Delta_{n}(x) \leqslant C(1)
$$

можно заменить на невозрастающую функцию $C_{N}(x)$ от $x \geqslant 0$ такую, что

$$
\frac{\sqrt{n}}{\beta_{3}} \sup _{|t| \geqslant x}|t|^{3} \Delta_{n}(t) \leqslant C_{N}(x) .
$$

В статье В.Н. Никулина [48] вычислены значения функции $C_{N}(x)$ при некоторых $x \geqslant 3.18$ (см. табл. 2). Несмотря на то, что использованный в [48] алгоритм подсчета $C_{N}(x)$ при больших $x$ дает лишь оценку $\lim _{x \rightarrow \infty} C_{N}(x) \leqslant 1+e$ (равномерную по $n \geqslant 1$ и $F \in \mathscr{F}_{3}$ ), там же при каждом фиксированном $n \geqslant 1$ и $F \in \mathscr{F}_{3}$ было аналитически исследовано предельное поведение $C_{N}(x)$ при $x \rightarrow \infty$ и показано, что для такой ситуации $\lim _{x \rightarrow \infty} C_{N}(x)=1$, т.е. $\sup _{n \geqslant 1, F \in \mathscr{F}_{3}} \lim \sup _{|x| \rightarrow \infty}|x|^{3} \Delta_{n}(x) / L_{n}^{3} \leqslant 1$.

Таблица 2. Значения функции $C_{N}(x)$ из работы [48].

\begin{tabular}{||c|c||c|c||c|c||}
\hline$x$ & $C_{N}(x)$ & $x$ & $C_{N}(x)$ & $x$ & $C_{N}(x)$ \\
\hline 3.18 & 28.4057 & 7.00 & 9.0590 & 20.00 & 4.9095 \\
4.00 & 22.1853 & 8.00 & 7.2512 & 50.00 & 4.2732 \\
5.00 & 16.0240 & 9.00 & 6.0329 & 100.00 & 4.0200 \\
6.00 & 11.8046 & 10.00 & 5.7370 & $\infty$ & $1+e$ \\
\hline
\end{tabular}

В 1953 г. А. Н. Колмогоров [7] ввел в рассмотрение функции

$$
D_{*}(x)=\limsup _{\ell \rightarrow 0} \sup _{n \geqslant 1,} \Delta_{F \in \mathscr{F}_{3}: L_{n}^{3}=\ell} \Delta_{n}(x) / \ell, \quad D^{*}(x)=\sup _{n \geqslant 1, F \in \mathscr{F}_{3}} \Delta_{n}(x) / L_{n}^{3}
$$


и поставил вопрос о возможно более точной оценке функций $D_{*}(x), D^{*}(x)$ (получение точных или асимптотически точных оценок). В 1990 г. Г. П. Чистяков $[27]$ показал, что

$$
\lim _{|x| \rightarrow \infty}|x|^{3} D_{*}(x)=1,
$$

откуда вытекает, что $\lim _{|x| \rightarrow \infty}|x|^{3} D^{*}(x) \geqslant 1$, поскольку $D_{*}(x) \leqslant D^{*}(x)$, $x \in \mathbf{R}$. Однако задача о построении точных или асимптотически точных оценок функции $D^{*}(x)$ оставалась нерешенной. В данной работе заполняются некоторые пробелы, связанные с изучением асимптотического поведения функции $D^{*}(x)$ и построением асимптотически точных мажорирующих функций для $D^{*}(x)$ и $D_{*}(x)$ при всех $0<\delta \leqslant 1$.

Введем аналоги правильной и асимптотически правильной постоянной в классическом (равномерном) неравенстве Берри-Эссеена для неравномерных оценок и $0<\delta \leqslant 1$ :

$$
\begin{aligned}
& C_{*}(x, \delta)=\limsup _{\ell \rightarrow 0} \sup _{n \geqslant 1, F \in \mathscr{F}_{2+\delta}: L_{n}^{2+\delta}=\ell} \sup _{|t| \geqslant x}|t|^{2+\delta} \Delta_{n}(t) / \ell, \quad x \geqslant 0, \\
& C^{*}(x, \delta)=\sup _{n \geqslant 1, F \in \mathscr{F}_{2+\delta}} \sup _{|t| \geqslant x}|t|^{2+\delta} \Delta_{n}(t) / L_{n}^{2+\delta}, \quad x \geqslant 0 .
\end{aligned}
$$

Тогда $\sup _{|t| \geqslant x}|t|^{3} D_{*}(t) \leqslant C_{*}(x, 1), \sup _{|t| \geqslant x}|t|^{3} D^{*}(t) \leqslant C^{*}(x, 1)$ для всех $x \geqslant 0$, а также с учетом результата работы Чистякова [27] $\lim _{x \rightarrow \infty} C_{*}(x, 1) \geqslant 1$.

Отметим, что с использованием функции $C_{N}(x)$ величины $C_{*}(x, 1)$ и $C^{*}(x, 1)$ при каждом $x \geqslant 0$ можно оценить следующим образом: $C_{*}(x, 1) \leqslant$ $C^{*}(x, 1) \leqslant C_{N}(x)$ (см. таблицу 2 ). Однако для предельного при $x \rightarrow \infty$ значения $C^{*}(x, 1)$ из работы [48] вытекает лишь оценка $\lim _{x \rightarrow \infty} C^{*}(x, 1) \leqslant$ $1+e$, поскольку асимптотика $C_{N}(x) \rightarrow 1$ при $x \rightarrow \infty$, доказанная в [48], не является равномерной по $n \geqslant 1$ и $F \in \mathscr{F}_{3}$.

В данной работе мы опишем алгоритм, позволяющий при каждом $0<\delta \leqslant 1$ строить ограниченную невозрастающую мажоранту $C(x, \delta)$ функции $C^{*}(x, \delta)$, т.е. такую функцию, что при каждом $x \geqslant 0$ справедливо неравенство

$$
\sup _{|t| \geqslant x}|t|^{2+\delta} \Delta_{n}(t) \leqslant C(x, \delta) L_{n}^{2+\delta}, \quad n \geqslant 1, \quad F \in \mathscr{F}_{2+\delta}
$$

(см. теорему 4). При этом $C(x, \delta)=C(\delta)-C_{0}(\delta)$ при $0 \leqslant x \leqslant K_{0}(\delta)$, $C(x, \delta)<C(0, \delta)$ при $x>K_{0}(\delta)$ (значения констант $K_{0}(\delta)$ будут указаны ниже) и $C(x, \delta) \rightarrow 1$ при $x \rightarrow \infty$, т.е. функция $C(x, 1)$ является асимптотически точной (неулучшаемой при $x \rightarrow \infty$ ) мажорантой для функций $C_{*}(x, 1)$ и $C^{*}(x, 1)$. Таким образом, оценка $(6)$ с указанной функцией $C(x, \delta)$ дополняет и обобщает результат работы Чистякова [27]: для 
асимптотического значения функции Колмогорова $D^{*}(x)$ получаем

$$
\limsup _{|x| \rightarrow \infty}|x|^{3} D^{*}(x)=1,
$$

а также $\lim _{x \rightarrow \infty} C_{*}(x, \delta) \leqslant \lim _{x \rightarrow \infty} C^{*}(x, \delta) \leqslant 1$ для всех $0<\delta \leqslant 1$.

Значения функции $C(x, 1)$ для $\delta=1$ и некоторых $x \geqslant K_{0}(1)$ и $x=0$ приведены в табл. 3 . Несложно убедиться, что $C(x, 1) \leqslant C_{N}(x)$, т.е. для $\delta=1$ неравенство (6) также уточняет результат работы [48]. При $0<\delta<1$ неравенство (6) приводится впервые.

Таблица 3. Значения функции $C(x, 1)$ в $(6)$ из теоремы 4.

\begin{tabular}{||c|c||c|c||c|c||}
\hline$x$ & $C(x, 1)$ & $x$ & $C(x, 1)$ & $x$ & $C(x, 1)$ \\
\hline 0 & 17.6355 & 3.8 & 15.3061 & 50 & 1.4388 \\
3.5 & 16.4933 & 4.0 & 14.5837 & 100 & 1.1565 \\
3.6 & 16.0383 & 10.0 & 4.6334 & 1000 & 1.0131 \\
3.7 & 15.6706 & 20.0 & 2.3099 & $\infty$ & 1 \\
\hline
\end{tabular}

Поскольку $\sup _{x \in \mathbf{R}}|x|^{2+\delta} \Delta_{n}(x) \leqslant C(0, \delta)$, из неравенства (6) легко получить следующую верхнюю оценку константы $C(\delta)$ в $(4): C(\delta) \leqslant$ $C(0, \delta)+C_{0}(\delta)$ (см. следствие 1$)$. Результаты вычислений для $\delta=$ $0.1,0.2, \ldots, 1$, проведенных в соответствии с алгоритмом построения мажоранты $C(\delta)$, описываемым в данной работе, приведены в табл. 4.

Таблица 4. Оценки констант $C(\delta)$ в (4) из следствия 1.

\begin{tabular}{||c|c||c|c||}
\hline$\delta=$ & $C(\delta) \leqslant$ & $\delta=$ & $C(\delta) \leqslant$ \\
\hline 1.0 & 18.1139 & 0.5 & 13.0258 \\
0.9 & 17.2651 & 0.4 & 11.9605 \\
0.8 & 16.1524 & 0.3 & 10.9675 \\
0.7 & 15.0866 & 0.2 & 10.0561 \\
0.6 & 14.0576 & 0.1 & 9.2114 \\
\hline
\end{tabular}

Идея, лежащая в основе описанного Л. Падитцем [58] конструктивного метода построения неравномерных оценок точности нормальной аппроксимации для распределений сумм независимых случайных величин, позволяющего получить явные оценки абсолютных констант, заключается в подходящем разбиении вещественной прямой на зоны «малых», «умеренных» и «больших» значений $x$. Традиционно используются разбиения вида

(i) («малые» значения $x$ ): $0 \leqslant x^{2} \leqslant K^{2}$;

(ii) («умеренные» значения $x$ ): $K^{2} \leqslant x^{2} \leqslant c_{n}(x ; \delta, a, b, c)$;

(iii) («большие» значения $x$ ): $c_{n}(x ; \delta, a, b, c) \leqslant x^{2}<\infty$, 
где $K>0, a>0, b>c \geqslant 1$ - вспомогательные свободные параметры, $c_{n}(x ; \delta, a, b, c)$ - некоторая функция, монотонно возрастающая по $x$ (см., в частности, [59], [58]). Положим

$$
c_{n}(x ; \delta, a, b, c)=\frac{b^{2}}{2(b-c)}\left[\ln |x|^{2+\delta}-\ln \left(a L_{n}^{2+\delta}\right)\right] \equiv \frac{b^{2}}{2(b-c)} \ln \frac{|x|^{2+\delta} n^{\delta / 2}}{a \beta_{2+\delta}} .
$$

Здесь мы развиваем методы и идеи, изложенные в [58] и [48]. Поскольку в некоторых работах, использующих эти методы, имеются пробелы, все утверждения, формулируемые в данной статье, снабжены подробными доказательствами.

2. Вспомогательные утверждения. Метод доказательства основан на усечении случайной величины $X_{1}$ :

$$
\bar{X}_{1}= \begin{cases}X_{1}, & \text { если }\left|X_{1}\right| \leqslant y, \\ 0 & \text { в противном случае }\end{cases}
$$

где $y>0$ - параметр усечения. Обозначим

$$
F_{n}^{y}(x)=\mathbf{P}\left(\sum_{k=1}^{n} \bar{X}_{k}<x\right) .
$$

Также для параметра $h \geqslant 0$ введем следующее обозначение:

$$
f(h)=\mathbf{E} e^{h \bar{X}_{1}}=\mathbf{E} \exp \left\{h X_{1} \mathbf{1}\left(\left|X_{1}\right| \leqslant y\right)\right\}, \quad x \in \mathbf{R} .
$$

Сформулируем и докажем несколько вспомогательных утверждений.

Лемма 1. Для произвольных параметров $h \geqslant 0$ и $y>0$ справедливьг неравенства

$$
\begin{gathered}
h-\frac{\beta_{2+\delta}}{y^{1+\delta}}\left(1+h y+\frac{(h y)^{2}}{2}\right) \leqslant m_{1} \equiv \mathbf{E} \bar{X}_{1} e^{h \bar{X}_{1}} \leqslant h+\frac{\beta_{2+\delta}}{y^{1+\delta}} e^{h y} \\
1-\frac{\beta_{2+\delta}}{y^{\delta}}(1+h y) \leqslant m_{2} \equiv \mathbf{E} \bar{X}_{1}^{2} e^{h \bar{X}_{1}} \leqslant 1+\frac{\beta_{2+\delta}}{y^{\delta}} e^{h y} \\
m_{3} \equiv \mathbf{E}\left|\bar{X}_{1}\right|^{3} e^{h \bar{X}_{1}} \leqslant \beta_{2+\delta} y^{1-\delta} e^{h y} \\
f(h) \equiv \mathbf{E} e^{h \bar{X}_{1}} \leqslant 1+\frac{h^{2}}{2}+\frac{\beta_{2+\delta} e^{h y}}{y^{2+\delta}} \leqslant \exp \left\{\frac{h^{2}}{2}+\frac{\beta_{2+\delta}}{y^{2+\delta}} e^{h y}\right\} \\
f(h) \geqslant 1-\frac{h \beta_{2+\delta}}{y^{1+\delta}} .
\end{gathered}
$$

Д о к а з а т е л ь с т в о. Доказательство основано на применении формулы Маклорена

$$
e^{h \bar{X}_{1}}=1+h \bar{X}_{1}+\frac{\left(h \bar{X}_{1}\right)^{2}}{2}+\sum_{k=3}^{\infty} \frac{\left(h \bar{X}_{1}\right)^{k}}{k !} .
$$


Найдем двусторонние оценки моментов случайной величины $\bar{X}_{1}$. Имеем

$$
\begin{aligned}
\left|\mathbf{E} \bar{X}_{1}\right| & =\left|\mathbf{E} X_{1}-\mathbf{E} X_{1} \mathbf{1}\left(\left|X_{1}\right|>y\right)\right| \leqslant \mathbf{E}\left|X_{1}\right| \mathbf{1}\left(\left|X_{1}\right|>y\right) \\
& \leqslant y^{-1-\delta} \mathbf{E}\left|X_{1}\right|^{2+\delta} \mathbf{1}\left(\left|X_{1}\right|>y\right) \leqslant \beta_{2+\delta} / y^{1+\delta}, \\
\mathbf{E} \bar{X}_{1}^{2}-1 & =\mathbf{E}\left(\bar{X}_{1}^{2}-X_{1}^{2}\right) \\
& =-\mathbf{E} X_{1}^{2} \mathbf{1}\left(\left|X_{1}\right|>y\right)\left\{\begin{array}{l}
\leqslant 0, \\
\geqslant-y^{-\delta} \mathbf{E}\left|X_{1}\right|^{2+\delta} \mathbf{1}\left(\left|X_{1}\right|>y\right) \geqslant-\beta_{2+\delta} / y^{\delta} .
\end{array}\right.
\end{aligned}
$$

$\left|\mathbf{E}\left(\bar{X}_{1}\right)^{k}\right| \leqslant \mathbf{E}\left|\bar{X}_{1}\right|^{k}=\mathbf{E}\left|X_{1}\right|^{k} \mathbf{1}\left(\left|X_{1}\right| \leqslant y\right) \leqslant \beta_{2+\delta} y^{k-2-\delta}, k \geqslant 3$.

Отсюда с использованием верхних оценок получаем

$$
\begin{aligned}
& m_{1} \equiv \mathbf{E} \bar{X}_{1} e^{h \bar{X}_{1}}=\mathbf{E}\left(\bar{X}_{1}\left(1+h \bar{X}_{1}+\sum_{k=2}^{\infty} \frac{\left(h \bar{X}_{1}\right)^{k}}{k !}\right)\right) \\
& =\mathbf{E} \bar{X}_{1}+h \mathbf{E} \bar{X}_{1}^{2}+\sum_{k=2}^{\infty} \frac{h^{k} \mathbf{E} \bar{X}_{1}^{k+1}}{k !} \leqslant \frac{\beta_{2+\delta}}{y^{1+\delta}}+h+\frac{\beta_{2+\delta}}{y^{1+\delta}} \sum_{k=2}^{\infty} \frac{(h y)^{k}}{k !} \\
& =\frac{\beta_{2+\delta}}{y^{1+\delta}}+h+\frac{\beta_{2+\delta}}{y^{1+\delta}}\left(e^{h y}-1-h y\right)=h+\frac{\beta_{2+\delta}}{y^{1+\delta}}\left(e^{h y}-h y\right) \text {, } \\
& m_{2} \equiv \mathbf{E} \bar{X}_{1}^{2} e^{h \bar{X}_{1}}=\mathbf{E}\left(\bar{X}_{1}^{2}\left(1+\sum_{k=1}^{\infty} \frac{\left(h \bar{X}_{1}\right)^{k}}{k !}\right)\right) \\
& =\mathbf{E} \bar{X}_{1}^{2}+\sum_{k=1}^{\infty} \frac{h^{k} \mathbf{E} \bar{X}_{1}^{k+2}}{k !} \leqslant 1+\frac{\beta_{2+\delta}}{y^{\delta}} \sum_{k=1}^{\infty} \frac{(h y)^{k}}{k !}=1+\frac{\beta_{2+\delta}}{y^{\delta}}\left(e^{h y}-1\right) . \\
& m_{3} \equiv \mathbf{E}\left|\bar{X}_{1}\right|^{3} e^{h \bar{X}_{1}} \leqslant \sum_{k=0}^{\infty} \frac{h^{k} \mathbf{E}\left|\bar{X}_{1}\right|^{k+3}}{k !} \leqslant \beta_{2+\delta} y^{1-\delta} \sum_{k=0}^{\infty} \frac{(h y)^{k}}{k !}=\beta_{2+\delta} y^{1-\delta} e^{h y} \\
& f(h) \equiv \mathbf{E} e^{h \bar{X}_{1}} \leqslant 1+h \mathbf{E} \bar{X}_{1}+\frac{\left(h \mathbf{E} \bar{X}_{1}\right)^{2}}{2}+\sum_{k=3}^{\infty} \frac{h^{k} \mathbf{E}\left|\bar{X}_{1}\right|^{k}}{k !} \\
& \leqslant 1+\frac{h \beta_{2+\delta}}{y^{1+\delta}}+\frac{h^{2}}{2}+\frac{\beta_{2+\delta}}{y^{2+\delta}} \sum_{k=3}^{\infty} \frac{(h y)^{k}}{k !} \\
& =1+\frac{h \beta_{2+\delta}}{y^{1+\delta}}+\frac{h^{2}}{2}+\frac{\beta_{2+\delta}}{y^{2+\delta}}\left(e^{h y}-1-h y-\frac{h^{2} y^{2}}{2}\right) \\
& =1+\frac{h^{2}}{2}+\frac{\beta_{2+\delta}}{y^{2+\delta}}\left(e^{h y}-1-\frac{h^{2} y^{2}}{2}\right) \text {. }
\end{aligned}
$$

Для доказательства (7) дополнительно заметим, что $x e^{h x} \geqslant x(1+h x+$ $\left.(h x)^{2} / 2\right), x \in \mathbf{R}$, и тогда получим оценку

$$
\begin{aligned}
m_{1} \equiv \mathbf{E} \bar{X}_{1} e^{h \bar{X}_{1}} & \geqslant \mathbf{E}\left(\bar{X}_{1}\left(1+h \bar{X}_{1}+0.5\left(h \bar{X}_{1}\right)^{2}\right)\right) \\
& \geqslant-\frac{\beta_{2+\delta}}{y^{1+\delta}}+h\left(1-\frac{\beta_{2+\delta}}{y^{\delta}}\right)-0.5 h^{2} \beta_{2+\delta} y^{1-\delta} \\
& =h-\frac{\beta_{2+\delta}}{y^{1+\delta}}\left(1+h y+0.5(h y)^{2}\right) .
\end{aligned}
$$


Замечая, что $e^{h x} \geqslant 1+h x, x \in \mathbf{R}$, получаем нижние оценки для $m_{2}$ и $f(h)$ :

$$
\begin{aligned}
m_{2} \equiv \mathbf{E} \bar{X}_{1}^{2} e^{h \bar{X}_{1}} & \geqslant \mathbf{E}\left(\bar{X}_{1}^{2}\left(1+h \bar{X}_{1}\right)\right) \\
& \geqslant 1-\frac{\beta_{2+\delta}}{y^{\delta}}-\beta_{2+\delta} h y^{1-\delta}=1-\frac{\beta_{2+\delta}}{y^{\delta}}(1+h y), \\
f(h) & \equiv \mathbf{E} e^{h \bar{X}_{1}} \geqslant \mathbf{E}\left(1+h \bar{X}_{1}\right) \geqslant 1-\frac{h \beta_{2+\delta}}{y^{1+\delta}}
\end{aligned}
$$

Лемма 1 доказана.

Лемма 2. Для параметра усечения у >0 справедливо неравенство

$$
\sup _{x \in \mathbf{R}}\left|F_{n}^{y}(x)-F_{n}(x)\right| \leqslant n \mathbf{P}\left(\left|X_{1}\right|>y\right) .
$$

Д о к а з а т е л ь с т в о. Замечая, что для любого $y>0$ события $\cap_{k=1}^{n}\left\{\left|X_{k}\right| \leqslant y\right\}$ и $\bigcup_{k=1}^{n}\left\{\left|X_{k}\right|>y\right\}$ образуют полную группу, для любого $x \in \mathbf{R}$ получаем

$$
\begin{aligned}
\left|F_{n}^{y}(x)-F_{n}(x)\right| & \mid \mathbf{P}\left(\sum_{k=1}^{n} \bar{X}_{k}<x, \bigcap_{k=1}^{n}\left\{\left|X_{k}\right| \leqslant y\right\}\right)+\mathbf{P}\left(\sum_{k=1}^{n} \bar{X}_{k}<x, \bigcup_{k=1}^{n}\left\{\left|X_{k}\right|>y\right\}\right) \\
& -\mathbf{P}\left(\sum_{k=1}^{n} X_{k}<x, \bigcap_{k=1}^{n}\left\{\left|X_{k}\right| \leqslant y\right\}\right)-\mathbf{P}\left(\sum_{k=1}^{n} X_{k}<x, \bigcup_{k=1}^{n}\left\{\left|X_{k}\right|>y\right\}\right) \mid \\
= & \left|\mathbf{P}\left(\sum_{k=1}^{n} \bar{X}_{k}<x, \bigcup_{k=1}^{n}\left\{\left|X_{k}\right|>y\right\}\right)-\mathbf{P}\left(\sum_{k=1}^{n} X_{k}<x, \bigcup_{k=1}^{n}\left\{\left|X_{k}\right|>y\right\}\right)\right| \\
\leqslant & \max \left\{\mathbf{P}\left(\sum_{k=1}^{n} \bar{X}_{k}<x, \bigcup_{k=1}^{n}\left\{\left|X_{k}\right|>y\right\}\right), \mathbf{P}\left(\sum_{k=1}^{n} X_{k}<x, \bigcup_{k=1}^{n}\left\{\left|X_{k}\right|>y\right\}\right)\right\} \\
\leqslant & \mathbf{P}\left(\bigcup_{k=1}^{n}\left\{\left|X_{k}\right|>y\right\}\right) \leqslant \sum_{k=1}^{n} \mathbf{P}\left(\left|X_{k}\right|>y\right)=n \mathbf{P}\left(\left|X_{1}\right|>y\right) .
\end{aligned}
$$

Лемма 3. $1^{\circ}$. Пусть $q>0 u A>0$. Тогда

$$
\sup _{v \geqslant A}|\Phi(v)-\Phi(q v)| \leqslant\left.\frac{1}{2} \max \left\{q^{2}-1, \frac{1-q^{2}}{q^{2}}\right\} \cdot(v \varphi(v))\right|_{v=\max \{1, A \min \{1, q\}\}} .
$$

$2^{\circ}$. Пусть $a \in \mathbf{R} u A>0$. Тогда

$$
\sup _{v \geqslant A}|\Phi(v+a)-\Phi(v)| \leqslant|a| \varphi(\min \{A+a, A\}) .
$$

Д о к а з а т е л ь с т в о. $1^{\circ}$. Пользуясь формулой Лагранжа, получаем

$$
\sup _{v \geqslant A}|\Phi(v)-\Phi(q v)| \leqslant|q-1| \sup _{v \geqslant A} v \varphi(v \cdot \min \{1, q\})
$$




$$
\begin{aligned}
& =\frac{|q-1|}{\min \{1, q\}} \sup _{v \geqslant A}(v \min \{1, q\} \varphi(v \min \{1, q\})) \\
& =|q-1| \max \left\{1, \frac{1}{q}\right\} \sup \{s \varphi(s): s \geqslant A \min \{1, q\}\} .
\end{aligned}
$$

Как несложно убедиться, для любого $q>0$

$$
|q-1| \max \left\{1, \frac{1}{q}\right\}=\max \left\{q-1, \frac{1-q}{q}\right\} \leqslant \frac{1}{2} \max \left\{q^{2}-1, \frac{1-q^{2}}{q^{2}}\right\} .
$$

Замечание о том, что функция $s \varphi(s), s \geqslant 0$, монотонно возрастает при $0<s<1$ и монотонно убывает при $s>1$, а следовательно, ее максимальное значение при $s \geqslant A \min \{1, q\}$ достигается в точке $s=\max \{1, A \min \{1, q\}\}$, завершает доказательство пункта $1^{\circ}$.

$2^{\circ}$. Также применяя формулу Лагранжа, имеем

$$
\sup _{v \geqslant A}|\Phi(v+a)-\Phi(v)| \leqslant|a| \sup _{v \geqslant A} \varphi(\min \{v+a, v\}) \leqslant|a| \varphi(\min \{A+a, A\}) .
$$

Лемма 4 (см. [3]). Для любой функиии распределения $F$ с нулевым средним и единичной дисперсией

$$
\sup _{x \in \mathbf{R}}|F(x)-\Phi(x)| \leqslant \sup _{x>0}\left(\Phi(x)-\frac{x^{2}}{1+x^{2}}\right)=0.54093654 \ldots \equiv \varkappa .
$$

Лемма 5. Для любой случайной величинь $X$ c $\mathbf{E}|X|^{3}<\infty u$ $\mathbf{E} X=а$ при любом а справедливо неравенство

$$
\begin{gathered}
\mathbf{E}|X-a|^{3} \leqslant \min \left\{L \cdot \mathbf{E}|X|^{3}, \mathbf{E}|X|^{3}+3|a| \mathbf{E} X^{2}+a^{2} \mathbf{E}|X|-|a|^{3}\right\} \\
L=\frac{17+7 \sqrt{7}}{27}<1.3156
\end{gathered}
$$

причем равенство $\mathbf{E}|X-a|^{3}=L \mathbf{E}|X|^{3}$ достигается на двухточечном распределении вида

$$
\mathbf{P}\left(X=\frac{6 a}{4-\sqrt{7} \pm \sqrt{1+2 \sqrt{7}}}\right)=\frac{3 \pm \sqrt{1+2 \sqrt{7}}}{6} .
$$

Д о к а з а т ель с т в о. Имеем

$$
\begin{aligned}
\mathbf{E}|X-a|^{3} & =\mathbf{E}|X-a|(X-a)^{2} \leqslant \mathbf{E}(|X|+|a|)\left(X^{2}-2 a X+a^{2}\right) \\
& =\mathbf{E}|X|^{3}-2 a \mathbf{E}(X|X|)+a^{2} \mathbf{E}|X|+|a| \mathbf{E} X^{2}-2|a| a \mathbf{E} X+|a|^{3} \\
& \leqslant \mathbf{E}|X|^{3}+2|a| \mathbf{E} X^{2}+a^{2} \mathbf{E}|X|+|a| \mathbf{E} X^{2}-|a|^{3},
\end{aligned}
$$

т.е. $\mathbf{E}|X-a|^{3}$ не превосходит второго аргумента минимума. Докажем, что $\mathbf{E}|X-a|^{3}$ не превосходит и первого аргумента минимума, 
т.е. $\mathbf{E}|X-a|^{3} \leqslant L \mathbf{E}|X|^{3}$. Для этого рассмотрим функционал $J_{a}(X)=$ $\mathbf{E}|X-a|^{3}-L \mathbf{E}|X|^{3}$ и покажем, что $\sup _{a} \sup _{X: \mathbf{E} X=a} J_{a}(X)=0$. Из результатов В.Хёффдинга [37] и В.М. Золотарева [6] вытекает, что экстремум линейного по функции распределения случайной величины $X$ функционала $J_{a}(X)$ с одним линейным ограничением $\mathbf{E} X=a$ моментного типа достигается на некотором двухточечном распределении. Пусть $\mathbf{P}(X=x)=p, \mathbf{P}(X=y)=q=1-p, 0<p<1$. Тогда $a=\mathbf{E} X=p x+q y, \mathbf{E}|X|^{3}=p|x|^{3}+q|y|^{3}, \mathbf{E}|X-a|^{3}=p q\left(p^{2}+q^{2}\right)|x-y|^{3}$. Обозначив $z=y / x$, получаем

$$
\sup _{X} \frac{\mathbf{E}|X-\mathbf{E} X|^{3}}{\mathbf{E}|X|^{3}}=\sup _{0<p<1, z \in \mathbf{R}} f(z, p), \quad \text { где } f(z, p)=p q\left(p^{2}+q^{2}\right) \frac{|1-z|^{3}}{p+q|z|^{3}} .
$$

Раскроем модули и покажем, что в каждом случае $f(z, p) \leqslant L$.

1) Пусть $z<0$. Тогда $f(z, p)=p q\left(p^{2}+q^{2}\right)(1-z)^{3} /\left(p-q z^{3}\right)$. Уравнение $f_{z}^{\prime}(z, p)=0$ равносильно $q z^{2}=p$, откуда находим, что $z=-\sqrt{p / q}$. Имеем

$$
f\left(-\sqrt{\frac{p}{q}}, p\right)=\left(p^{2}+q^{2}\right)(\sqrt{p}+\sqrt{q})^{2}=(1-2 p q)(1+2 \sqrt{p q}) .
$$

Положим $s=\sqrt{p q}$ и рассмотрим функцию $g(s)=\left(1-2 s^{2}\right)(1+2 s)$, $s>0$. Она достигает своего максимального значения $(17+7 \sqrt{7}) / 27=$ $1.315565 \ldots$ в точке $s=(\sqrt{7}-1) / 6 \approx 0.2743$, т.е. экстремальное распределение $X$ задается параметрами

$$
\begin{gathered}
p=\frac{3-\sqrt{1+2 \sqrt{7}}, \quad q=\frac{3+\sqrt{1+2 \sqrt{7}}}{6},}{6}, \\
\frac{y}{x}=-\sqrt{\frac{4-\sqrt{7}}{2+\sqrt{7}+3 \sqrt{1+2 \sqrt{7}}}} .
\end{gathered}
$$

2) Пусть $0 \leqslant z \leqslant 1$. Тогда $f(z, p)=p q\left(p^{2}+q^{2}\right)(1-z)^{3} /\left(p+q z^{3}\right)$. $\mathrm{C}$ точностью до неотрицательного множителя производная $f_{z}^{\prime}(z, p)$ имеет вид $-3(1-z)^{2}\left(p+q z^{2}\right)$, откуда заключаем, что $f_{z}^{\prime}(z, p) \leqslant 0$ при $0 \leqslant z \leqslant 1$, а следовательно, максимальное значение функции $f(z, p)$ на отрезке $0 \leqslant z \leqslant 1$ достигается в точке $z=0$ и имеет вид $f(0, p)=q\left(p^{2}+q^{2}\right)=$ $(1-p)\left(1-2 p+2 p^{2}\right)$. Несложно убедиться, что $f_{p}^{\prime}(0, p)<0$ для всех $p \in \mathbf{R}$, а следовательно, $\sup _{0<p<1} f(0, p)=\lim _{p \rightarrow 0+} f(0, p)=1<L$.

3) Пусть $z>1$. Тогда $f(z, p)=p q\left(p^{2}+q^{2}\right)(z-1)^{3} /\left(p+q z^{3}\right)$. С точностью до неотрицательного множителя производная $f_{z}^{\prime}(z, p)$ имеет вид $3(z-1)^{2}\left(p+q z^{2}\right)$, откуда получаем, что $f_{z}^{\prime}(z, p) \geqslant 0$ при $z>1$, а следовательно, $\sup _{z>1} f(z, p)=\lim _{z \rightarrow \infty} f(z, p)=p\left(p^{2}+q^{2}\right)$. Аналогично случаю 2$)$ заключаем, что и здесь $f(z, p)$ не превосходит 1 . Лемма доказана. 
3. Случаи (i) и (iii) - «малые» и «большие» значения $x$. В случае (i), т.е. для $0 \leqslant|x| \leqslant K$, в соответствии с неравенством (1) мы имеем

$$
|x|^{2+\delta} \Delta_{n}(x) \leqslant C_{0}(\delta) K^{2+\delta} L_{n}^{2+\delta}
$$

В случае же (iii), т.е. для

$$
x^{2} \geqslant \frac{b^{2}}{2(b-c)} \ln \frac{|x|^{2+\delta} n^{\delta / 2}}{a \beta_{2+\delta}}
$$

справедлив следующий результат.

Теорема 1. Предположим, ито $c_{n}(x ; \delta, a, b, c)>0, x^{2} \geqslant \max \left\{(2 \pi)^{-1}\right.$, $\left.c_{n}(x ; \delta, a, b, c)\right\}, b>c \geqslant 1, a>0$. Тогда для любого $n \geqslant 1 u F \in \mathscr{F}_{2+\delta}$

$$
|x|^{2+\delta} \Delta_{n}(x) \leqslant P(\delta, a, b, c, x) L_{n}^{2+\delta}
$$

әдe

$$
P(\delta, a, b, c, x)=b^{2+\delta}+a \exp \left\{\frac{b^{2+\delta}}{a}-\frac{2(b-c)(c-1)}{b^{2}} x^{2}\right\} .
$$

3 а м е ч а н и е 1 . Очевидно, функция $P(\delta, a, b, c, x)$ монотонно не возрастает по $|x|$. Также несложно убедиться, что функция $P(\delta, a, b, c, x)$ аргумента $a>0$ при фиксированных $\delta, b, c$ и $x$ достигает своего минимального значения в точке $a=b^{2+\delta}$ :

$$
\inf _{a>0} P(\delta, a, b, c, x)=b^{2+\delta}\left(1+\exp \left\{1-\frac{2(b-c)(c-1)}{b^{2}} x^{2}\right\}\right)
$$

при этом $P(\delta, a, b, c, x) \geqslant 1$ для всех рассматриваемых значений $\delta, a, b, c, x$. Кроме того, для любого $0<\delta \leqslant 1$

$$
\inf _{a>0, b>c \geqslant 1} \lim _{|x| \rightarrow \infty} P(\delta, a, b, c, x)=\lim _{b \rightarrow 1+} b^{2+\delta}=1 .
$$

Д ок а з а т е л с т в о т е о р е м ы 1. Без ограничения общности пусть $x \geqslant 0$. Поскольку

$$
\Delta_{n}(x)=\max \left\{\left(1-F_{n}(x \sqrt{n})\right)+(\Phi(x)-1),\left(F_{n}(x \sqrt{n})-1\right)+(1-\Phi(x))\right\},
$$

и величины $F_{n}(x \sqrt{n})-1$ и $\Phi(x)-1$ неположительны для любого $t \in \mathbf{R}$, получаем

$$
\Delta_{n}(x) \leqslant \max \left\{1-F_{n}(x \sqrt{n}), 1-\Phi(x)\right\} .
$$

Для всех $x \geqslant \max \left\{(2 \pi)^{-1 / 2}, \sqrt{c_{n}(x ; \delta, a, b, c)}\right\}$ справедливо неравенство

$$
1-\Phi(x) \leqslant \frac{\varphi(x)}{x}=\frac{e^{-x^{2} / 2}}{x \sqrt{2 \pi}} \leqslant e^{-x^{2} / 2} \leqslant\left[\frac{a \beta_{2+\delta}}{x^{2+\delta} n^{\delta / 2}}\right]^{b^{2} /(4(b-c))} .
$$


Замечая, что $b^{2} /(4(b-c)) \geqslant c$ при $b \geqslant c$ и для рассматриваемого диапазона значений $x$

$$
\frac{a \beta_{2+\delta}}{x^{2+\delta} n^{\delta / 2}} \leqslant \exp \left\{-\frac{2(b-c) x^{2}}{b^{2}}\right\}<1,
$$

окончательно заключаем, что

$$
1-\Phi(x) \leqslant\left[\frac{a \beta_{2+\delta}}{x^{2+\delta} n^{\delta / 2}}\right]^{c} .
$$

С другой стороны, для $1-F_{n}(x \sqrt{n})$ мы имеем

$$
1-F_{n}(x \sqrt{n}) \leqslant 1-F_{n}^{y}(x \sqrt{n})+\left|F_{n}^{y}(x \sqrt{n})-F_{n}(x \sqrt{n})\right|
$$

с параметром усечения $y=x \sqrt{n} / b, b>1$. Тогда по лемме 2 и по неравенству Маркова получим

$$
\left|F_{n}^{y}(x \sqrt{n})-F_{n}(x \sqrt{n})\right| \leqslant n \mathbf{P}\left(\left|X_{1}\right|>y\right) \leqslant \frac{b^{2+\delta} \beta_{2+\delta}}{x^{2+\delta} n^{\delta / 2}} .
$$

Обозначим

$$
h=\frac{1}{y} \ln \frac{n^{\delta / 2} x^{2+\delta}}{\beta_{2+\delta} a} \equiv \frac{2(b-c)}{b x \sqrt{n}} c_{n}(x ; \delta, a, b, c) .
$$

Используя неравенство Маркова и учитывая верхнюю оценку (10) для $f(h)=\mathbf{E} e^{h \bar{X}_{1}}$ из леммы 1 для указанного $h$, получаем

$$
\begin{aligned}
1 & -F_{n}^{y}(x \sqrt{n})=\mathbf{P}\left(h \bar{X}_{1}+\cdots+h \bar{X}_{n} \geqslant h x \sqrt{n}\right) \leqslant f^{n}(h) \exp \{-h x \sqrt{n}\} \\
& \leqslant \exp \left\{-h x \sqrt{n}+\frac{1}{2} h^{2} n+\frac{n \beta_{2+\delta}}{y^{2+\delta}} e^{h y}\right\} \\
& =\exp \left\{-\frac{2(b-c)}{b} c_{n}(x ; \delta, a, b, c)+\frac{2(b-c)^{2}}{b^{2} x^{2}} c_{n}^{2}(x ; \delta, a, b, c)+\frac{b^{2+\delta}}{a}\right\} .
\end{aligned}
$$

Для $x^{2} \geqslant c_{n}(x ; \delta, a, b, c)$ имеем

$$
\begin{aligned}
1-F_{n}^{y}(x \sqrt{n}) & \leqslant \exp \left\{-\frac{2 c(b-c)}{b^{2}} c_{n}(x ; \delta, a, b, c)+\frac{b^{2+\delta}}{a}\right\} \\
& =\left(\frac{a \beta_{2+\delta}}{x^{2+\delta} n^{\delta / 2}}\right)^{c} \exp \left\{\frac{b^{2+\delta}}{a}\right\},
\end{aligned}
$$

откуда видно, что мажоранта для $1-F_{n}(x \sqrt{n})$ не меньше, чем мажоранта для $1-\Phi(x)$. Далее, учитывая (14), получаем

$$
1-F_{n}^{y}(x \sqrt{n}) \leqslant a \exp \left\{\frac{b^{2+\delta}}{a}-\frac{2(b-c)(c-1)}{b^{2}} x^{2}\right\} \frac{\beta_{2+\delta}}{x^{2+\delta} n^{\delta / 2}} .
$$

Суммируя оценки (15) и (16), приходим к (13). Теорема 1 доказана. 
4. Случай (ii) - «умеренные» значения $x$. Переопределим величины

$$
y=\gamma x \sqrt{n}, \quad h=\frac{(1-\gamma) x}{\sqrt{n}}, \quad \gamma \in(0,1) .
$$

Обозначим $S_{n}^{*}=X_{1}^{*}+\cdots+X_{n}^{*}$ сумму независимых одинаково распределенных случайных величин с общей функцией распределения

$$
\mathbf{P}\left(X_{1}^{*}<u\right)=\frac{1}{f(h)} \int_{-\infty}^{u} e^{h t} d \mathbf{P}\left(\bar{X}_{1}<t\right) .
$$

Легко проверить справедливость следующих равенств:

$$
\begin{aligned}
1-\Phi(x) & =\exp \left\{\frac{h^{2} n}{2}\right\} \int_{x}^{+\infty} e^{-h \sqrt{n} t} d \Phi(t-h \sqrt{n}), \\
1-F_{n}^{y}(x \sqrt{n}) & =f^{n}(h) \int_{x}^{+\infty} e^{-h \sqrt{n} t} d \mathbf{P}\left(S_{n}^{*}<t \sqrt{n}\right) .
\end{aligned}
$$

Пусть без ограничения общности $x \geqslant 0$. Тогда

$$
\begin{aligned}
\Delta_{n}(x) & =\left|1-\Phi(x)+\left(F_{n}(x \sqrt{n})-F_{n}^{y}(x \sqrt{n})\right)+F_{n}^{y}(x \sqrt{n})-1\right| \\
& \leqslant\left|F_{n}(x \sqrt{n})-F_{n}^{y}(x \sqrt{n})\right|+\left|1-\Phi(x)+F_{n}^{y}(x \sqrt{n})-1\right| .
\end{aligned}
$$

Далее воспользуемся неравенством леммы 2 и выражениями $(17),(18)$ :

$$
\begin{aligned}
\Delta_{n}(x) \leqslant & n \mathbf{P}\left(\left|X_{1}\right|>y\right) \\
& +\left|\left(\exp \left\{\frac{h^{2} n}{2}\right\}-f^{n}(h)\right) \int_{x}^{+\infty} e^{-h \sqrt{n} t} d \mathbf{P}\left(S_{n}^{*}<t \sqrt{n}\right)\right| \\
& +\left|\exp \left\{\frac{h^{2} n}{2}\right\} \int_{x}^{+\infty} e^{-h \sqrt{n} t} d\left(\Phi(t-h \sqrt{n})-\mathbf{P}\left(S_{n}^{*}<t \sqrt{n}\right)\right)\right| .
\end{aligned}
$$

Применяя неравенство Маркова к первому слагаемому и интегрируя по частям последнее, получаем

$$
\begin{aligned}
\Delta_{n}(x) \leqslant & \frac{\beta_{2+\delta}}{\gamma^{2+\delta} x^{2+\delta} n^{\delta / 2}}+\left|f^{n}(h)-e^{h^{2} n / 2}\right| \exp \{-h x \sqrt{n}\} \cdot \mathbf{P}\left(S_{n}^{*}>x \sqrt{n}\right) \\
& +2 \exp \left\{\frac{h^{2} n}{2}-h x \sqrt{n}\right\} \cdot \sup _{u \geqslant x}\left|\mathbf{P}\left(S_{n}^{*}<u \sqrt{n}\right)-\Phi(u-h \sqrt{n})\right| \\
\equiv & \frac{\beta_{2+\delta}}{\gamma^{2+\delta} x^{2+\delta} n^{\delta / 2}}+I_{1} \cdot I_{2}+2 \exp \left\{\frac{h^{2} n}{2}-h x \sqrt{n}\right\} \cdot I_{3}
\end{aligned}
$$

Сформулируем два утверждения, которые будут неоднократно использоваться в дальнейшем. Во-первых, если $x^{2} \leqslant c_{n}(x ; \delta, a, b, c)$, то (см. (ii))

$$
L_{n}^{2+\delta} \leqslant \frac{x^{2+\delta}}{a} \exp \left\{-\frac{2(b-c)}{b^{2}} x^{2}\right\}
$$


а следовательно,

$$
\begin{gathered}
\frac{1}{n} \leqslant\left(L_{n}^{2+\delta}\right)^{2 / \delta} \leqslant \frac{x^{2+4 / \delta}}{a^{2 / \delta}} \exp \left\{-\frac{4(b-c)}{\delta b^{2}} x^{2}\right\} \\
\frac{1}{\sqrt{n}} \leqslant\left(L_{n}^{2+\delta}\right)^{1 / \delta} \leqslant \frac{x^{1+2 / \delta}}{a^{1 / \delta}} \exp \left\{-\frac{2(b-c)}{\delta b^{2}} x^{2}\right\} \\
n^{\delta / 2-1} \leqslant\left(L_{n}^{2+\delta}\right)^{(2-\delta) / \delta} \leqslant \frac{x^{(2+\delta)(2-\delta) / \delta}}{a^{(2-\delta) / \delta}} \exp \left\{-\frac{2(2-\delta)(b-c)}{\delta b^{2}} x^{2}\right\}, \\
n^{(\delta-1) / 2} \leqslant\left(L_{n}^{2+\delta}\right)^{(1-\delta) / \delta} \leqslant \frac{x^{(2+\delta)(1-\delta) / \delta}}{a^{(1-\delta) / \delta}} \exp \left\{-\frac{2(1-\delta)(b-c)}{\delta b^{2}} x^{2}\right\} .
\end{gathered}
$$

Во-вторых, если $x^{2} \geqslant K^{2}$, то при $x \geqslant \sqrt{r /(2 s)}, r>0$, или при $r \leqslant 0$

$$
x^{r} \exp \left\{-s x^{2}\right\} \leqslant K^{r} \exp \left\{-s K^{2}\right\}, \quad s>0 .
$$

Всюду далее мы будем предполагать, что параметры $b>c \geqslant 1$, $\gamma \in(0,1), K>0$ удовлетворяют следующим условиям:

$$
\begin{aligned}
\frac{2(b-c)}{b^{2}} & >\gamma(1-\gamma) \\
K^{2} & \geqslant \frac{b^{2}}{b-c}, \\
K^{2} & \geqslant \frac{4+5 \delta}{2 \delta}\left[\frac{2(2+\delta)(b-c)}{\delta b^{2}}-\gamma(1-\gamma)\right]^{-1}, \\
K^{2} & \geqslant \frac{4+5 \delta}{4 \delta}\left[\frac{2(1+\delta)(b-c)}{\delta b^{2}}-\gamma(1-\gamma)\right]^{-1} \\
K^{2} & \geqslant \frac{3}{2}\left[\frac{2(b-c)}{b^{2}}-\gamma(1-\gamma)\right]^{-1}, \\
K^{2} & \geqslant \frac{4+3 \delta}{2 \delta}\left[\frac{2(2+\delta)(b-c)}{\delta b^{2}}-2 \gamma(1-\gamma)\right]^{-1} \\
K^{2} & \geqslant \frac{2(1-\delta)}{\delta}\left[\frac{4(b-c)}{\delta b^{2}}-\gamma(1-\gamma)\right]^{-1} \\
K^{2} & \geqslant \frac{3}{(1-\gamma)^{2}}, \\
K^{2} & \geqslant \frac{4}{1-\gamma^{2}} .
\end{aligned}
$$

4.1. Оценка $I_{1}$. Оценим выражение

$$
I_{1}=\left|f^{n}(h)-\exp \left\{\frac{n h^{2}}{2}\right\}\right| \exp \{-h x \sqrt{n}\} .
$$

Применяя оценку (10) из леммы 1 и неравенство $\left|e^{x}-1\right| \leqslant|x| e^{|x|}$, получим

$$
\left|f^{n}(h)-\exp \left\{\frac{n h^{2}}{2}\right\}\right| \leqslant \exp \left\{\frac{h^{2} n}{2}\right\}\left|\exp \left\{\frac{n \beta_{2+\delta} e^{h y}}{y^{2+\delta}}\right\}-1\right|
$$




$$
\begin{aligned}
& \leqslant \frac{n \beta_{2+\delta}}{y^{2+\delta}} \exp \left\{\frac{n \beta_{2+\delta} e^{h y}}{y^{2+\delta}}+h y+\frac{h^{2} n}{2}\right\} \\
& =\frac{\beta_{2+\delta}}{(\gamma x)^{2+\delta} n^{\delta / 2}} \exp \left\{\frac{\beta_{2+\delta} e^{\gamma(1-\gamma) x^{2}}}{(\gamma x)^{2+\delta} n^{\delta / 2}}+\frac{\left(1-\gamma^{2}\right) x^{2}}{2}\right\} .
\end{aligned}
$$

Воспользуемся оценкой (20):

$$
\left|f^{n}(h)-\exp \left\{\frac{n h^{2}}{2}\right\}\right| \leqslant \frac{\beta_{2+\delta}}{(\gamma x)^{2+\delta} n^{\delta / 2}} \exp \left\{\frac{\left(1-\gamma^{2}\right) x^{2}}{2}\right\} A_{1}(x)
$$

где

$$
\ln A_{1}(x) \equiv \frac{1}{a \gamma^{2+\delta}} \exp \left\{-\left(\frac{2(b-c)}{b^{2}}-\gamma(1-\gamma)\right) x^{2}\right\} \leqslant \ln A_{1}(K),
$$

при $x \geqslant K$, если выполнено условие (26). Здесь и далее символами $A(x), A_{1}(x), A_{2}(x), \ldots$ будут обозначаться положительные функции аргумента $x$, также зависящие от $\delta$ и параметров $a, b, c, \gamma$. Таким образом,

$$
\begin{aligned}
I_{1} & \leqslant \frac{1}{\gamma^{2+\delta}} \exp \left\{-(1-\gamma) x^{2}+\left(1-\gamma^{2}\right) x^{2} / 2\right\} A_{1}(x) \frac{\beta_{2+\delta}}{x^{2+\delta} n^{\delta / 2}} \\
& \leqslant A_{1}(K) A_{2}(K) \frac{\beta_{2+\delta}}{x^{2+\delta} n^{\delta / 2}},
\end{aligned}
$$

где $A_{2}(x)=\gamma^{-(2+\delta)} \exp \left\{-(1-\gamma)^{2} x^{2} / 2\right\} \leqslant A_{2}(K)$ при $x \geqslant K$.

4.2. Оценка $I_{2}$. Оценим выражение $I_{2} \equiv \mathbf{P}\left(S_{n}^{*}>x \sqrt{n}\right)$. С учетом (2) мы получаем

$$
\begin{aligned}
I_{2} \leqslant & \left|\mathbf{P}\left(S_{n}^{*}<x \sqrt{n}\right)-\Phi\left(\frac{x \sqrt{n}-\mathbf{E} S_{n}^{*}}{\sqrt{\mathbf{D} S_{n}^{*}}}\right)\right|+\Phi\left(-\frac{x \sqrt{n}-\mathbf{E} S_{n}^{*}}{\sqrt{\mathbf{D} S_{n}^{*}}}\right) \\
& \leqslant 0.3041 \frac{\mathbf{E}\left|X_{1}^{*}-\mathbf{E} X_{1}^{*}\right|^{3}}{\sqrt{n}\left(\mathbf{D} X_{1}^{*}\right)^{3 / 2}}+\frac{0.3041}{\sqrt{n}}+\Phi\left(-\frac{x \sqrt{n}-\mathbf{E} S_{n}^{*}}{\sqrt{\mathbf{D} S_{n}^{*}}}\right) .
\end{aligned}
$$

Формально вместо первых двух слагаемых последнего выражения можно поставить минимум из $0.3041\left(\mathbf{E}\left|X_{1}^{*}-\mathbf{E} X_{1}^{*}\right|^{3} /\left(\mathbf{D} X_{1}^{*}\right)^{3 / 2}+1\right) / \sqrt{n}$ и $0.33477\left(\mathbf{E}\left|X_{1}^{*}-\mathbf{E} X_{1}^{*}\right|^{3} /\left(\mathbf{D} X_{1}^{*}\right)^{3 / 2}+0.429\right) / \sqrt{n}$ (обе оценки получены в [12], [40]), однако, как показывают вычисления, в экстремальном случае этот минимум достигается на той оценке, у которой абсолютная константа при ляпуновской дроби меньше (т.е. на оценке (2)), поэтому для краткости всюду далее мы будем использовать лишь оценку (2).

Для удобства дальнейших ссылок заметим, что в силу оценок (11), (20) и $(21)$

$$
\begin{aligned}
f(h) & \geqslant 1-\frac{h \beta_{2+\delta}}{y^{1+\delta}}=1-\frac{(1-\gamma) \beta_{2+\delta}}{\gamma^{1+\delta} x^{\delta} n^{1+\delta / 2}} \\
& \geqslant 1-\frac{(1-\gamma) x^{4+4 / \delta}}{a^{1+2 / \delta} \gamma^{1+\delta}} \exp \left\{-\frac{2(2+\delta)(b-c)}{\delta b^{2}} x^{2}\right\} \\
& \equiv A_{3}(x) \geqslant A_{3}(K),
\end{aligned}
$$


если, в соответствии с $(25), K^{2} \geqslant(1+\delta) /(2+\delta) \cdot b^{2} /(b-c)$, а это условие вытекает из (27). Теперь оценим $\left(\mathbf{E} X_{1}^{*}\right)^{2}=\left(m_{1} / f(h)\right)^{2}$. С учетом (7), (20) и $(22)$ имеем

$$
\mathbf{E}\left(X_{1}^{*}\right)^{2} \leqslant \frac{1}{A_{3}^{2}(K)}\left(h+\frac{\beta_{2+\delta} e^{h y}}{y^{1+\delta}}\right)^{2} \leqslant A_{4}(x),
$$

где

$$
\begin{aligned}
A_{4}(x)=\frac{1}{A_{3}^{2}(K)}[ & \frac{(1-\gamma) x^{2+2 / \delta}}{a^{1 / \delta}} \exp \left\{-\frac{2(b-c)}{\delta b^{2}} x^{2}\right\} \\
& \left.+\frac{x^{2+2 / \delta}}{a^{1+1 / \delta} \gamma^{1+\delta}} \exp \left\{-\left(\frac{2(1+\delta)(b-c)}{\delta b^{2}}-\gamma(1-\gamma)\right) x^{2}\right\}\right]^{2} .
\end{aligned}
$$

В соответствии с $(25)$ заключаем, что $A_{4}(x) \leqslant A_{4}(K)$, если выполнены условия

$$
K^{2} \geqslant \frac{(1+\delta) b^{2}}{2(b-c)}, \quad K^{2} \geqslant \frac{(1+\delta)}{\delta}\left[\frac{2(1+\delta)(b-c)}{\delta b^{2}}-\gamma(1-\gamma)\right]^{-1},
$$

вытекающие соответственно из (27) и (29). Далее, с учетом (10) имеем

$$
(f(h))^{-1} \geqslant 2-f(h) \geqslant 1-\frac{h^{2}}{2}-\frac{\beta_{2+\delta} e^{h y}}{y^{2+\delta}} \geqslant 1-A_{5}(x),
$$

где

$$
\begin{aligned}
A_{5}(x)= & \frac{(1-\gamma)^{2} x^{4+4 / \delta}}{2 a^{2 / \delta}} \exp \left\{-\frac{4(b-c) x^{2}}{\delta b^{2}}\right\} \\
& +\frac{x^{2+4 / \delta}}{a^{1+2 / \delta} \gamma^{2+\delta}} \exp \left\{-\left(\frac{2(2+\delta)(b-c)}{\delta b^{2}}-\gamma(1-\gamma)\right) x^{2}\right\} .
\end{aligned}
$$

При этом $A_{5}(x) \leqslant A_{5}(K)$, если справедливы более сильные условия $(27)$ и (28). Таким образом, используя нижнюю оценку (8) для $m_{2}=$ $f(h) \mathbf{E}\left(X_{1}^{*}\right)^{2}$ и неравенства $(35),(20)$, получаем

$$
\begin{aligned}
\mathbf{D} X_{1}^{*} & =\mathbf{E}\left(X_{1}^{*}\right)^{2}-\left(\mathbf{E} X_{1}^{*}\right)^{2} \geqslant \frac{1}{f(h)}-\frac{\beta_{2+\delta}}{f(h) y^{\delta}}(1+h y)-\left(\mathbf{E} X_{1}^{*}\right)^{2} \\
& \geqslant 1-A_{5}(K)-\frac{1}{A_{3}(K)} \cdot \frac{\beta_{2+\delta}}{(\gamma x)^{\delta} n^{\delta / 2}}\left(1+\gamma(1-\gamma) x^{2}\right)-A_{4}(K) \\
& \geqslant 1-A_{6}(x)
\end{aligned}
$$

где

$$
A_{6}(x)=A_{4}(K)+A_{5}(K)+\frac{1}{A_{3}(K)} \cdot \frac{x^{2}}{a \gamma^{\delta}}\left(1+\gamma(1-\gamma) x^{2}\right) \exp \left\{-\frac{2(b-c) x^{2}}{b^{2}}\right\} .
$$


При этом $A_{6}(x) \leqslant A_{6}(K)$ в силу (27). Таким образом,

$$
\mathbf{D} X_{1}^{*} \geqslant 1-A_{6}(K) \equiv A_{7}(K) .
$$

Из леммы 5 получаем

$$
\begin{aligned}
\mathbf{E}\left|X_{1}^{*}-\mathbf{E} X_{1}^{*}\right|^{3} \leqslant \min \{ & L \mathbf{E}\left|X_{1}^{*}\right|^{3}, \mathbf{E}\left|X_{1}^{*}\right|^{3}+3 \mathbf{E}\left(X_{1}^{*}\right)^{2}\left|\mathbf{E} X_{1}^{*}\right| \\
& \left.+\mathbf{E}\left|X_{1}^{*}\right|\left(\mathbf{E} X_{1}^{*}\right)^{2}\right\} .
\end{aligned}
$$

Оценим входящие в правую часть моменты по лемме 1. Имеем

$$
\begin{aligned}
& \mathbf{E}\left|X_{1}^{*}\right|^{3}=\frac{m_{3}}{f(h)} \leqslant \frac{y^{1-\delta} e^{h y} \beta_{2+\delta}}{A_{3}(K)} \\
& =\frac{(\gamma x)^{1-\delta}}{A_{3}(K)} \exp \left\{\gamma(1-\gamma) x^{2}\right\} n^{(1-\delta) / 2} \beta_{2+\delta} \\
& \leqslant \frac{\sqrt{n}}{A_{3}(K)} \cdot \frac{\gamma^{1-\delta} x^{3}}{a} \exp \left\{-\left(\frac{2(b-c)}{b^{2}}-\gamma(1-\gamma)\right) x^{2}\right\} \\
& 3 \mathbf{E}\left(X_{1}^{*}\right)^{2}\left|\mathbf{E} X_{1}^{*}\right|=3 \frac{m_{2}}{f(h)} \cdot \frac{m_{1}}{f(h)} \leqslant \frac{3}{A_{3}^{2}(K)}\left(1+\frac{\beta_{2+\delta} e^{h y}}{y^{\delta}}\right)\left(h+\frac{\beta_{2+\delta} e^{h y}}{y^{1+\delta}}\right) \\
& =\frac{3 \sqrt{n}}{A_{3}^{2}(K)}\left[\frac{(1-\gamma) x}{n}+\frac{\beta_{2+\delta} e^{\gamma(1-\gamma) x^{2}}}{(\gamma x)^{1+\delta} n^{1+\delta / 2}}\right. \\
& \left.+\frac{\beta_{2+\delta} e^{\gamma(1-\gamma) x^{2}}}{(\gamma x)^{\delta} n^{\delta / 2}} \cdot \frac{(1-\gamma) x}{n}+\frac{\beta_{2+\delta}^{2} e^{2 \gamma(1-\gamma) x^{2}}}{(\gamma x)^{1+2 \delta} n^{1+\delta}}\right] \\
& \leqslant \frac{3 \sqrt{n}}{A_{3}^{2}(K)}\left[\frac{(1-\gamma) x^{3+4 / \delta}}{a^{2 / \delta}} \exp \left\{-\frac{4(b-c) x^{2}}{\delta b^{2}}\right\}\right. \\
& +\frac{x^{3+4 / \delta}}{a^{1+2 / \delta} \gamma^{1+\delta}} \exp \left\{-\left(\frac{2(2+\delta)(b-c)}{\delta b^{2}}-\gamma(1-\gamma)\right) x^{2}\right\} \\
& +\frac{(1-\gamma) x^{5+4 / \delta}}{a^{1+2 / \delta} \gamma^{\delta}} \exp \left\{-\left(\frac{2(2+\delta)(b-c)}{\delta b^{2}}-\gamma(1-\gamma)\right) x^{2}\right\} \\
& \left.+\frac{x^{5+4 / \delta}}{a^{2+2 / \delta} \gamma^{1+2 \delta}} \exp \left\{-\left(\frac{4(1+\delta)(b-c)}{\delta b^{2}}-2 \gamma(1-\gamma)\right) x^{2}\right\}\right] \\
& \equiv A_{8}(x) \sqrt{n}, \\
& \mathbf{E}\left|X_{1}^{*}\right|\left(\mathbf{E} X_{1}^{*}\right)^{2} \leqslant \sqrt{\mathbf{E}\left(X_{1}^{*}\right)^{2}}\left(\mathbf{E} X_{1}^{*}\right)^{2} \leqslant\left(\frac{m_{2}}{f(h)}\right)^{1 / 2} \cdot\left(\frac{m_{1}}{f(h)}\right)^{2} \\
& \leqslant \frac{1}{A_{3}^{5 / 2}(K)}\left(1+\frac{\beta_{2+\delta} e^{h y}}{y^{\delta}}\right)^{1 / 2} \cdot\left(h+\frac{\beta_{2+\delta} e^{h y}}{y^{1+\delta}}\right)^{2} \\
& =\frac{\sqrt{n}}{A_{3}^{5 / 2}(K)}\left(\frac{1}{n}+\frac{\beta_{2+\delta} e^{\gamma(1-\gamma) x^{2}}}{(\gamma x)^{\delta} n^{1+\delta / 2}}\right)^{1 / 2} \cdot\left(\frac{(1-\gamma) x}{\sqrt{n}}+\frac{\beta_{2+\delta} e^{\gamma(1-\gamma) x^{2}}}{(\gamma x)^{1+\delta} n^{(1+\delta) / 2}}\right)^{2}
\end{aligned}
$$




$$
\begin{aligned}
& \leqslant \frac{\sqrt{n}}{A_{3}^{5 / 2}(K)}\left(\frac{x^{2+4 / \delta}}{a^{2 / \delta}} \exp \left\{-\frac{4(b-c) x^{2}}{\delta b^{2}}\right\}\right. \\
& \left.\quad+\frac{x^{4+4 / \delta}}{a^{1+2 / \delta} \gamma^{\delta}} \exp \left\{-\left(\frac{2(2+\delta)(b-c)}{\delta b^{2}}-\gamma(1-\gamma)\right) x^{2}\right\}\right)^{1 / 2} \\
& \quad \times\left(\frac{(1-\gamma) x^{2+2 / \delta}}{a^{1 / \delta}} \exp \left\{-\frac{2(b-c)}{\delta b^{2}} x^{2}\right\}\right. \\
& \left.\quad+\frac{x^{2+2 / \delta}}{a^{1+1 / \delta} \gamma^{1+\delta}} \exp \left\{-\left(\frac{2(1+\delta)(b-c)}{\delta b^{2}}-\gamma(1-\gamma)\right) x^{2}\right\}\right)^{2} \\
& \equiv A_{9}(x) \sqrt{n} .
\end{aligned}
$$

Таким образом,

$$
\mathbf{E}\left|X_{1}^{*}-\mathbf{E} X_{1}^{*}\right|^{3} \leqslant A_{10}(x) \sqrt{n}
$$

где

$$
\begin{aligned}
& A_{10}(x)=\min \left\{\frac{L \gamma^{1-\delta} x^{3}}{a A_{3}(K)} e^{-\left(2(b-c) / b^{2}-\gamma(1-\gamma)\right) x^{2}},\right. \\
&\left.\frac{\gamma^{1-\delta} x^{3}}{a A_{3}(K)} e^{-\left(2(b-c) / b^{2}-\gamma(1-\gamma)\right) x^{2}}+A_{8}(x)+A_{9}(x)\right\} .
\end{aligned}
$$

В соответствии с $(25)$ заключаем, что $A_{10}(x) \leqslant A_{10}(K)$, если справедливо более сильное условие (27) и условия $(28),(29),(30)$. Следовательно, по неравенству (2) с учетом (37) и (38), а также с учетом (22), получаем

$$
\begin{aligned}
& \left|\mathbf{P}\left(\frac{S_{n}^{*}-\mathbf{E} S_{n}^{*}}{\sqrt{\mathbf{D} S_{n}^{*}}}<u\right)-\Phi(u)\right| \leqslant 0.3041 \frac{\mathbf{E}\left|X_{1}^{*}-\mathbf{E} X_{1}^{*}\right|^{3}}{\sqrt{n}\left(\mathbf{D} X_{1}^{*}\right)^{3 / 2}}+\frac{0.3041}{\sqrt{n}} \\
& \quad \leqslant 0.3041 \frac{A_{10}(K)}{A_{7}^{3 / 2}(K)}+0.3041 \frac{x^{1+2 / \delta}}{a^{1 / \delta}} \exp \left\{-\frac{2(b-c)}{\delta b^{2}} x^{2}\right\} .
\end{aligned}
$$

Теперь оценим величину

$$
u \equiv \frac{x \sqrt{n}-\mathbf{E} S_{n}^{*}}{\sqrt{\mathbf{D} S_{n}^{*}}}=\frac{x-\sqrt{n} \mathbf{E} X_{1}^{*}}{\sqrt{\mathbf{D} X_{1}^{*}}} .
$$

Используя верхнюю оценку (7) для $m_{1} \equiv \mathbf{E} \bar{X}_{1} e^{h \bar{X}_{1}}=f(h) \mathbf{E} X_{1}^{*}$ и нижние оценки $(11),(35)$ для $f(h)$, получаем

$$
\begin{aligned}
& \mathbf{E} X_{1}^{*}-h=\frac{m_{1}}{f(h)}-h \leqslant \frac{1}{f(h)}\left(h+\frac{\beta_{2+\delta} e^{h y}}{y^{1+\delta}}\right)-h=\frac{(1-f(h)) h}{f(h)}+\frac{\beta_{2+\delta} e^{h y}}{f(h) y^{1+\delta}} \\
& \leqslant \frac{h^{2} \beta_{2+\delta}}{A_{3}(K) y^{1+\delta}}+\frac{\beta_{2+\delta} e^{h y}}{A_{3}(K) y^{1+\delta}} \leqslant \frac{1}{\sqrt{n} A_{3}(K)}\left(\frac{(1-\gamma)^{2} x^{2} \beta_{2+\delta}}{(\gamma x)^{1+\delta} n^{\delta / 2+1}}+\frac{\beta_{2+\delta} e^{\gamma(1-\gamma) x^{2}}}{(\gamma x)^{1+\delta} n^{\delta / 2}}\right) \\
& \leqslant \frac{1}{\sqrt{n} A_{3}(K)}\left(\frac{(1-\gamma)^{2} x^{5+4 / \delta}}{a^{1+2 / \delta} \gamma^{1+\delta}} \exp \left\{-\frac{2(2+\delta)(b-c) x^{2}}{\delta b^{2}}\right\}\right.
\end{aligned}
$$




$$
\left.+\frac{x}{a \gamma^{1+\delta}} \exp \left\{-\left(\frac{2(b-c)}{b^{2}}-\gamma(1-\gamma)\right) x^{2}\right\}\right) \equiv \frac{A_{11}(x)}{\sqrt{n}}
$$

В соответствии с $(25)$ заключаем, что $A_{11}(x) \leqslant A_{11}(K)$ при $x \geqslant K$ в силу (27) и (30).

Далее нам понадобится следующая верхняя оценка для $\mathbf{D} X_{1}^{*}$, которую мы получим с учетом верхней оценки (8) для $m_{2}$ :

$$
\begin{aligned}
\mathbf{D} X_{1}^{*} & \leqslant \mathbf{E}\left(X_{1}^{*}\right)^{2}=\frac{m_{2}}{f(h)} \leqslant \frac{1}{A_{3}(K)}\left(1+\frac{e^{h y} \beta_{2+\delta}}{y^{\delta}}\right) \\
& \leqslant \frac{1}{A_{3}(K)}\left(1+\frac{e^{\gamma(1-\gamma) x^{2}} \beta_{2+\delta}}{(\gamma x)^{\delta} n^{\delta / 2}}\right) \\
& \leqslant \frac{1}{A_{3}(K)}\left(1+\frac{x^{2}}{a \gamma^{\delta}} \exp \left\{-\left(\frac{2(b-c)}{b^{2}}-\gamma(1-\gamma)\right) x^{2}\right\}\right) \\
& \equiv A_{13}(x) \leqslant A_{13}(K),
\end{aligned}
$$

если $K^{2} \geqslant\left(2(b-c) / b^{2}-\gamma(1-\gamma)\right)^{-1}$, что вытекает из $(30)$.

Найдем верхнюю оценку для $\sqrt{n} \mathbf{E} X_{1}^{*}-x$. Учитывая (40), получаем

$$
\begin{aligned}
\sqrt{n} \mathbf{E} X_{1}^{*}-x & =\sqrt{n} \mathbf{E} X_{1}^{*}-h \sqrt{n}+h \sqrt{n}-x=\sqrt{n}\left(\mathbf{E} X_{1}^{*}-h\right)-\gamma x \\
& \leqslant A_{11}(K)-\gamma K,
\end{aligned}
$$

т.е. при $x \geqslant K$

$$
\frac{x-\sqrt{n} \mathbf{E} X_{1}^{*}}{\sqrt{\mathbf{D} X_{1}^{*}}} \geqslant \frac{\gamma K-A_{11}(K)}{\sqrt{A_{13}(K)}} \equiv A_{14}(K) .
$$

Отсюда, учитывая также оценку (39) и условие (27), мы окончательно получаем

$$
\begin{aligned}
I_{2} \leqslant & \left|\mathbf{P}\left(S_{n}^{*}<x \sqrt{n}\right)-\Phi\left(\frac{x \sqrt{n}-\mathbf{E} S_{n}^{*}}{\sqrt{\mathbf{D} S_{n}^{*}}}\right)\right|+\Phi\left(-\frac{x-\sqrt{n} \mathbf{E} X_{1}^{*}}{\sqrt{\mathbf{D} X_{1}^{*}}}\right) \\
\leqslant & 0.3041 \frac{A_{10}(K)}{A_{7}^{3 / 2}(K)}+0.3041 \frac{K^{1+2 / \delta}}{a^{1 / \delta}} \exp \left\{-\frac{2(b-c)}{\delta b^{2}} K^{2}\right\} \\
& +\frac{1}{\sqrt{2 \pi} A_{14}(K)} \exp \left\{-\frac{A_{14}^{2}(K)}{2}\right\} \equiv A_{15}(K) .
\end{aligned}
$$

Оценка (42) справедлива при $A_{14}(K)>0$.

4.3. Оценка $I_{3}$. Приступим к оцениванию величины $I_{3} \equiv$ $\sup _{u \geqslant x}\left|\mathbf{P}\left(S_{n}^{*}<u \sqrt{n}\right)-\Phi(u-h \sqrt{n})\right|$. Имеем

$$
I_{3}=\sup _{u \geqslant x} \mid \mathbf{P}\left(\frac{S_{n}^{*}-\mathbf{E} S_{n}^{*}}{\sqrt{\mathbf{D} S_{n}^{*}}}<\frac{u \sqrt{n}-\mathbf{E} S_{n}^{*}}{\sqrt{\mathbf{D} S_{n}^{*}}}\right)-\Phi\left(\frac{u \sqrt{n}-\mathbf{E} S_{n}^{*}}{\sqrt{\mathbf{D} S_{n}^{*}}}\right)
$$




$$
\begin{aligned}
&+\Phi\left(\frac{u-\sqrt{n} \mathbf{E} X_{1}^{*}}{\sqrt{\mathbf{D} X_{1}^{*}}}\right)-\Phi\left(u-\sqrt{n} \mathbf{E} X_{1}^{*}\right) \\
&+\Phi\left(u-\sqrt{n} \mathbf{E} X_{1}^{*}\right)-\Phi(u-h \sqrt{n}) \mid \\
& \leqslant \sup _{v \in \mathbf{R}}\left|\mathbf{P}\left(\frac{S_{n}^{*}-\mathbf{E} S_{n}^{*}}{\sqrt{\mathbf{D} S_{n}^{*}}}<v\right)-\Phi(v)\right| \\
&+\sup _{v \geqslant\left(x-\sqrt{n} \mathbf{E} X_{1}^{*}\right) / \sqrt{\mathbf{D} X_{1}^{*}}}\left|\Phi(v)-\Phi\left(v \sqrt{\mathbf{D} X_{1}^{*}}\right)\right| \\
&+\sup _{u \geqslant x}\left|\Phi\left(u-\sqrt{n} \mathbf{E} X_{1}^{*}\right)-\Phi(u-h \sqrt{n})\right|=I_{31}+I_{32}+I_{33} .
\end{aligned}
$$

Для оценки $I_{31}$ воспользуемся неравенством (2), из которого получаем

$$
I_{31} \leqslant 0.3041 \frac{\mathbf{E}\left|X_{1}^{*}-\mathbf{E} X_{1}^{*}\right|^{3}}{\sqrt{n}\left(\mathbf{D} X_{1}^{*}\right)^{3 / 2}}+\frac{0.3041}{\sqrt{n}} .
$$

Величину $\mathbf{E}\left|X_{1}^{*}-\mathbf{E} X_{1}^{*}\right|^{3}$ оценим аналогично (38), только выделив множитель $n^{(1-\delta) / 2} \beta_{2+\delta}=L_{n}^{2+\delta} \sqrt{n}$. Имеем

$$
\begin{aligned}
& \mathbf{E}\left|X_{1}^{*}-\mathbf{E} X_{1}^{*}\right|^{3} \leqslant \min \left\{L \mathbf{E}\left|X_{1}^{*}\right|^{3}, \mathbf{E}\left|X_{1}^{*}\right|^{3}+3 \mathbf{E}\left(X_{1}^{*}\right)^{2}\left|\mathbf{E} X_{1}^{*}\right|\right. \\
& \left.+\mathbf{E}\left|X_{1}^{*}\right|\left(\mathbf{E} X_{1}^{*}\right)^{2}\right\} \\
& \mathbf{E}\left|X_{1}^{*}\right|^{3}=\frac{m_{3}}{f(h)} \leqslant \frac{y^{1-\delta} e^{h y} \beta_{2+\delta}}{A_{3}(K)}=\frac{(\gamma x)^{1-\delta}}{A_{3}(K)} \exp \left\{\gamma(1-\gamma) x^{2}\right\} n^{(1-\delta) / 2} \beta_{2+\delta}, \\
& 3 \mathbf{E}\left(X_{1}^{*}\right)^{2}\left|\mathbf{E} X_{1}^{*}\right|=3 \frac{m_{2}}{f(h)} \cdot \frac{m_{1}}{f(h)} \leqslant \frac{3}{A_{3}^{2}(K)}\left(1+\frac{\beta_{2+\delta} e^{h y}}{y^{\delta}}\right)\left(h+\frac{\beta_{2+\delta} e^{h y}}{y^{1+\delta}}\right) \\
& =\frac{3 \sqrt{n}}{A_{3}^{2}(K)}\left[\frac{(1-\gamma) x}{n}+\frac{\beta_{2+\delta} e^{\gamma(1-\gamma) x^{2}}}{(\gamma x)^{1+\delta} n^{1+\delta / 2}}+\frac{\beta_{2+\delta} e^{\gamma(1-\gamma) x^{2}}}{(\gamma x)^{\delta} n^{\delta / 2}} \cdot \frac{(1-\gamma) x}{n}\right. \\
& \left.+\frac{\beta_{2+\delta}^{2} e^{2 \gamma(1-\gamma) x^{2}}}{(\gamma x)^{1+2 \delta} n^{1+\delta}}\right] \\
& \leqslant \frac{3 n^{(1-\delta) / 2} \beta_{2+\delta}}{A_{3}^{2}(K)}\left[\frac{(1-\gamma) x^{(2+\delta)(2-\delta) / \delta+1}}{a^{(2-\delta) / \delta}} \exp \left\{-\frac{2(2-\delta)(b-c)}{\delta b^{2}} x^{2}\right\}\right. \\
& +\frac{x^{4 / \delta+1-\delta}+x^{4 / \delta+3-\delta} \gamma(1-\gamma)}{a^{2 / \delta} \gamma^{1+\delta}} \exp \left\{-\left(\frac{4(b-c)}{\delta b^{2}}-\gamma(1-\gamma)\right) x^{2}\right\} \\
& \left.+\frac{x^{4 / \delta+3-\delta}}{a^{1+2 / \delta} \gamma^{1+2 \delta}} \exp \left\{-\left(\frac{2(2+\delta)(b-c)}{\delta b^{2}}-2 \gamma(1-\gamma)\right) x^{2}\right\}\right] \\
& \equiv \widehat{A}_{8}(x) n^{(1-\delta) / 2} \beta_{2+\delta}, \\
& \mathbf{E}\left|X_{1}^{*}\right|\left(\mathbf{E} X_{1}^{*}\right)^{2} \leqslant \sqrt{\mathbf{E}\left(X_{1}^{*}\right)^{2}}\left(\mathbf{E} X_{1}^{*}\right)^{2}=\sqrt{m_{2} / f(h)} \cdot\left(\frac{m_{1}}{f(h)}\right)^{2} \\
& \leqslant \frac{1}{A_{3}^{5 / 2}(K)}\left(\left(1+\frac{\beta_{2+\delta} e^{h y}}{y^{\delta}}\right)^{1 / 2} \cdot\left(h+\frac{\beta_{2+\delta} e^{h y}}{y^{1+\delta}}\right)^{2}\right.
\end{aligned}
$$




$$
\begin{aligned}
= & \frac{n^{(1-\delta) / 2} \beta_{2+\delta}}{A_{3}^{5 / 2}(K)} n^{\delta / 2-1}\left(\frac{1}{n}+\frac{\beta_{2+\delta} e^{\gamma(1-\gamma) x^{2}}}{(\gamma x)^{\delta} n^{1+\delta / 2}}\right)^{1 / 2} \\
& \times\left((1-\gamma) x+\frac{\beta_{2+\delta} e^{\gamma(1-\gamma) x^{2}}}{(\gamma x)^{1+\delta} n^{\delta / 2}}\right)^{2} \\
\leqslant & \frac{n^{(1-\delta) / 2} \beta_{2+\delta} x^{(2+\delta)(2-\delta) / \delta}}{A_{3}^{5 / 2}(K) a^{(2-\delta) / \delta}} \exp \left\{-\frac{2(2-\delta)(b-c)}{\delta b^{2}} x^{2}\right\} \\
& \times\left[\frac{x^{4 / \delta+2}}{a^{2 / \delta}} \exp \left\{-\frac{4(b-c)}{\delta b^{2}} x^{2}\right\}+\frac{x^{4 / \delta+4}}{a^{1+2 / \delta} \gamma^{\delta}}\right. \\
& \left.\quad \times \exp \left\{-\left(\frac{2(2+\delta)(b-c)}{\delta b^{2}}-\gamma(1-\gamma)\right) x^{2}\right\}\right]^{1 / 2} \\
& \times\left[x(1-\gamma)+\frac{x}{a \gamma^{1+\delta}} \exp \left\{-\left(\frac{2(b-c)}{b^{2}}-\gamma(1-\gamma)\right) x^{2}\right\}\right]^{2} \\
\leqslant & \widehat{A}_{9}(x) n^{(1-\delta) / 2} \beta_{2+\delta},
\end{aligned}
$$

где

$$
\begin{aligned}
\widehat{A}_{9}(x)= & \frac{x^{(2+\delta)(2-\delta) / \delta+2}}{A_{3}^{5 / 2}(K) a^{(2-\delta) / \delta}} \exp \left\{-\frac{2(2-\delta)(b-c)}{\delta b^{2}} x^{2}\right\} \\
\times & {\left[\frac{K^{4 / \delta+2}}{a^{2 / \delta}} \exp \left\{-\frac{4(b-c)}{\delta b^{2}} K^{2}\right\}\right.} \\
& \left.\quad+\frac{K^{4 / \delta+4}}{a^{1+2 / \delta} \gamma^{\delta}} \exp \left\{-\left(\frac{2(2+\delta)(b-c)}{\delta b^{2}}-\gamma(1-\gamma)\right) K^{2}\right\}\right]^{1 / 2} \\
\times & {\left[1-\gamma+\frac{1}{a \gamma^{1+\delta}} \exp \left\{-\left(\frac{2(b-c)}{b^{2}}-\gamma(1-\gamma)\right) K^{2}\right\}\right]^{2} }
\end{aligned}
$$

в силу (26), (27) и (28). Таким образом, $\mathbf{E}\left|X_{1}^{*}-\mathbf{E} X_{1}^{*}\right|^{3} \leqslant$ $\widehat{A}_{10}(x) n^{(1-\delta) / 2} \beta_{2+\delta}$, где

$$
\widehat{A}_{10}(x)=\min \left\{\frac{L(\gamma x)^{1-\delta} e^{\gamma(1-\gamma) x^{2}}}{A_{3}(K)}, \frac{(\gamma x)^{1-\delta} e^{\gamma(1-\gamma) x^{2}}}{A_{3}(K)}+\widehat{A}_{8}(x)+\widehat{A}_{9}(x)\right\} .
$$

Воспользуемся неравенством (37), чтобы оценить величину $\mathbf{D} X_{1}^{*}$, неравенством $(24)$ и условием $(27)$, чтобы оценить $n^{(\delta-1) / 2}$. Тогда для $I_{31}$ окончательно получим

$$
\begin{aligned}
I_{31} & \leqslant 0.3041 \frac{\mathbf{E}\left|X_{1}^{*}-\mathbf{E} X_{1}^{*}\right|^{3}}{\sqrt{n}\left(\mathbf{D} X_{1}^{*}\right)^{3 / 2}}+\frac{0.3041}{\sqrt{n}} \\
& \leqslant 0.3041\left(\frac{\widehat{A}_{10}(x)}{A_{7}^{3 / 2}(K)}+\frac{K^{(2+\delta)(1-\delta) / \delta}}{a^{(1-\delta) / \delta}} \exp \left\{-\frac{2(1-\delta)(b-c)}{\delta b^{2}} K^{2}\right\}\right) \frac{\beta_{2+\delta}}{n^{\delta / 2}} .
\end{aligned}
$$


Для $I_{32}$ в силу (41) имеем

$$
\begin{aligned}
I_{32} & \equiv \sup _{v \geqslant\left(x-\sqrt{n} \mathbf{E} X_{1}^{*}\right) / \sqrt{\mathbf{D} X_{1}^{*}}}\left|\Phi(v)-\Phi\left(v \sqrt{\mathbf{D} X_{1}^{*}}\right)\right| \\
& \leqslant \sup _{v \geqslant A_{14}(K)}\left|\Phi(v)-\Phi\left(v \sqrt{\mathbf{D} X_{1}^{*}}\right)\right| .
\end{aligned}
$$

Используя утверждение $1^{\circ}$ леммы 3 в предположении, что $A_{14}(K)>0$, а также оценку $\min \left\{1, \sqrt{\mathbf{D} X_{1}^{*}}\right\} \geqslant \min \left\{1, \sqrt{A_{7}(K)}\right\}=\sqrt{A_{7}(K)}$ (см. (37)) и замечая, что функция $v \varphi(v)$ монотонно убывает при $v>1$, получаем

$$
I_{32} \leqslant\left.\frac{1}{2} \max \left\{\mathbf{D} X_{1}^{*}-1, \frac{1-\mathbf{D} X_{1}^{*}}{\mathbf{D} X_{1}^{*}}\right\} \cdot(v \varphi(v))\right|_{v=\max \left\{1, A_{14}(K) \sqrt{A_{7}(K)}\right\}} .
$$

Оценим $\mathbf{D} X_{1}^{*}$ (аналогично случаю $I_{2}$, только будем выделять ляпуновскую дробь $\left.\beta_{2+\delta} / n^{\delta / 2}\right)$. Во-первых,

$$
\begin{aligned}
\left(\mathbf{E} X_{1}^{*}\right)^{2} & \leqslant \frac{1}{A_{3}^{2}(K)}\left(h+\frac{\beta_{2+\delta} e^{h y}}{y^{1+\delta}}\right)^{2} \\
& =\frac{\beta_{2+\delta} n^{\delta / 2-1}}{n^{\delta / 2} A_{3}^{2}(K)}\left((1-\gamma) x+\frac{\beta_{2+\delta} e^{\gamma(1-\gamma) x^{2}}}{(x \gamma)^{1+\delta} n^{\delta / 2}}\right)^{2} \leqslant \widehat{A}_{4}(x) L_{n}^{2+\delta},
\end{aligned}
$$

где в силу (26)

$$
\begin{aligned}
\widehat{A}_{4}(x)= & \frac{x^{(2+\delta)(2-\delta) / \delta+2}}{A_{3}^{2}(K) a^{(2-\delta) / \delta}} \exp \left\{-\frac{2(2-\delta)(b-c)}{\delta b^{2}} x^{2}\right\} \\
& \times\left[(1-\gamma)+\frac{1}{a \gamma^{1+\delta}} \exp \left\{-\left(\frac{2(b-c)}{b^{2}}-\gamma(1-\gamma)\right) K^{2}\right\}\right]^{2} .
\end{aligned}
$$

Далее, по лемме 1 имеем

$$
\begin{aligned}
\frac{1}{f(h)} & \geqslant 2-f(h) \geqslant 1-\frac{h^{2}}{2}-\frac{\beta_{2+\delta} e^{h y}}{y^{2+\delta}} \geqslant 1-\left(\frac{(1-\gamma)^{2} x^{2}}{2 n^{1-\delta / 2}}+\frac{e^{\gamma(1-\gamma) x^{2}}}{(x \gamma)^{2+\delta} n}\right) L_{n}^{2+\delta} \\
& \geqslant 1-\widehat{A}_{5}(x) L_{n}^{2+\delta}
\end{aligned}
$$

где

$$
\begin{aligned}
\widehat{A}_{5}(x)= & \frac{(1-\gamma)^{2} x^{(2+\delta)(2-\delta) / \delta+2}}{2 a^{(2-\delta) / \delta}} \exp \left\{-\frac{2(2-\delta)(b-c)}{\delta b^{2}} x^{2}\right\} \\
& +\frac{x^{4 / \delta-\delta}}{a^{2 / \delta} \gamma^{2+\delta}} \exp \left\{-\left(\frac{4(b-c)}{\delta b^{2}}-\gamma(1-\gamma)\right) x^{2}\right\}
\end{aligned}
$$

а также

$$
m_{2}=f(h) \mathbf{E}\left(X_{1}^{*}\right)^{2} \geqslant 1-\frac{\beta_{2+\delta}}{y^{\delta}}(1+h y)=1-\frac{1+\gamma(1-\gamma) x^{2}}{(x \gamma)^{\delta}} L_{n}^{2+\delta} .
$$


Таким образом,

$$
\begin{aligned}
\mathbf{D} X_{1}^{*} & =\mathbf{E}\left(X_{1}^{*}\right)^{2}-\left(\mathbf{E} X_{1}^{*}\right)^{2} \geqslant \frac{1}{f(h)}-\frac{\beta_{2+\delta}}{f(h) y^{\delta}}(1+h y)-\left(\mathbf{E} X_{1}^{*}\right)^{2} \\
& \geqslant 1-\frac{\widehat{A}_{5}(x) \beta_{2+\delta}}{n^{\delta / 2}}-\frac{1}{A_{3}(K)(\gamma x)^{\delta}}\left(1+\gamma(1-\gamma) x^{2}\right) L_{n}^{2+\delta}-\widehat{A}_{4}(x) L_{n}^{2+\delta} \\
& =1-\widehat{A}_{6}(x) L_{n}^{2+\delta},
\end{aligned}
$$

где

$$
\widehat{A}_{6}(x)=\widehat{A}_{4}(x)+\widehat{A}_{5}(x)+\frac{1+\gamma(1-\gamma) x^{2}}{A_{3}(K)(\gamma x)^{\delta}}
$$

Замечая, что $\widehat{A}_{6}(x)>0$ и учитывая оценку $\mathbf{D} X_{1}^{*} \geqslant A_{7}(K)$ (см. (37)), получаем

$$
\frac{1-\mathbf{D} X_{1}^{*}}{\mathbf{D} X_{1}^{*}} \leqslant \frac{\widehat{A}_{6}(x)}{A_{7}(K)} L_{n}^{2+\delta}
$$

С другой стороны, в силу леммы 1

$$
\begin{aligned}
\mathbf{D} X_{1}^{*}-1 & \leqslant \frac{m_{2}-f(h)}{f(h)} \leqslant \frac{e^{h y} \beta_{2+\delta}}{f(h) y^{\delta}}+\frac{1-f(h)}{f(h)} \leqslant \frac{\beta_{2+\delta}}{f(h) y^{\delta}}\left(e^{h y}+\frac{h}{y}\right) \\
& \leqslant \frac{\beta_{2+\delta}}{n^{\delta / 2} A_{3}(K)(\gamma x)^{\delta}}\left[\exp \left\{\gamma(1-\gamma) x^{2}\right\}\right. \\
& \left.\quad+\frac{(1-\gamma) x^{4 / \delta+2}}{a^{2 / \delta} \gamma} \exp \left\{-\frac{4(b-c) x^{2}}{\delta b^{2}}\right\}\right] \leqslant A_{12}(x) \frac{\beta_{2+\delta}}{n^{\delta / 2}}
\end{aligned}
$$

где в силу (27)

$$
\begin{aligned}
A_{12}(x)=\frac{1}{\gamma^{\delta} A_{3}(K)}\left[x^{-\delta} \exp \left\{\gamma(1-\gamma) x^{2}\right\}+\right. & \frac{(1-\gamma) K^{4 / \delta-\delta+2}}{a^{2 / \delta} \gamma} \\
& \left.\times \exp \left\{-\frac{4(b-c) K^{2}}{\delta b^{2}}\right\}\right] .
\end{aligned}
$$

Таким образом, для $I_{32}$ окончательно получаем

$$
I_{32} \leqslant\left.\frac{1}{2} \max \left\{A_{12}(x), \frac{\widehat{A}_{6}(x)}{A_{7}(K)}\right\} L_{n}^{2+\delta} \cdot(v \varphi(v))\right|_{v=\max \left\{1, A_{14}(K) \sqrt{A_{7}(K)}\right\}} .
$$

Наконец, рассмотрим величину $I_{33}$ :

$$
\begin{aligned}
I_{33} & =\sup _{u \geqslant x}\left|\Phi\left(u-\mathbf{E} S_{n}^{*} / \sqrt{n}\right)-\Phi(u-h \sqrt{n})\right| \\
& =\sup _{v \geqslant x-h \sqrt{n}}\left|\Phi\left(v+\sqrt{n}\left(h-\mathbf{E} X_{1}^{*}\right)\right)-\Phi(v)\right| .
\end{aligned}
$$


Учитывая, что $x-h \sqrt{n}=x \gamma \geqslant \gamma K>0$, и пользуясь утверждением $2^{\circ}$ леммы 3 , получим

$$
I_{33} \leqslant\left|\sqrt{n}\left(\mathbf{E} X_{1}^{*}-h\right)\right| \varphi\left(\min \left\{\gamma K+\sqrt{n}\left(h-\mathbf{E} X_{1}^{*}\right), \gamma K\right\}\right) .
$$

Воспользуемся нижней оценкой (7) для $m_{1} \equiv \mathbf{E} \bar{X}_{1} e^{h \bar{X}_{1}}=f(h) \mathbf{E} X_{1}^{*}$ :

$$
\begin{aligned}
h-\mathbf{E} X_{1}^{*} & =h-\frac{m_{1}}{f(h)}=\frac{1}{f(h)}\left(h f(h)-m_{1}\right) \\
& \leqslant \frac{1}{f(h)}\left(h(f(h)-1)+\frac{\beta_{2+\delta}}{y^{1+\delta}}\left(1+h y+\frac{(h y)^{2}}{2}\right)\right) .
\end{aligned}
$$

Оценим выражение $h(f(h)-1)$ с помощью неравенства $(10)$ :

$$
\begin{aligned}
h(f(h)-1) \leqslant & \left(\frac{h^{2}}{2}+\frac{\beta_{2+\delta} e^{h y}}{y^{2+\delta}}\right) h=(h y)^{3} \frac{\beta_{2+\delta}}{y^{1+\delta}}\left(\frac{1}{2 y^{2-\delta}}+\frac{e^{h y}}{y^{4} h^{2}}\right) \\
= & (h y)^{3} \frac{\beta_{2+\delta}}{y^{1+\delta}}\left(\frac{n^{\delta / 2-1}}{2(\gamma x)^{2-\delta}}+\frac{e^{\gamma(1-\gamma) x^{2}}}{(\gamma x)^{4} n(1-\gamma)^{2} x^{2}}\right) \\
\leqslant & (h y)^{3} \frac{\beta_{2+\delta}}{y^{1+\delta}} A(x), \\
A(x) \equiv & \frac{x^{4 / \delta-2}}{2 a^{(2-\delta) / \delta} \gamma^{2-\delta}} \exp \left\{-\frac{2(2-\delta)(b-c) x^{2}}{\delta b^{2}}\right\} \\
& +\frac{x^{4 / \delta-4}}{a^{2 / \delta} \gamma^{4}(1-\gamma)^{2}} \exp \left\{-\left(\frac{4(b-c)}{\delta b^{2}}-\gamma(1-\gamma)\right) x^{2}\right\} .
\end{aligned}
$$

При этом $A(x) \leqslant A(K)$, при $x^{2} \geqslant K^{2}$, если выполнено более сильное условие (27) и условие (32). Далее, в предположении, что $A(K) \leqslant 1 / 6$, мы имеем

$$
\begin{aligned}
h-\mathbf{E} X_{1}^{*} & \leqslant \frac{1}{A_{3}(K)}\left(\frac{(h y)^{3}}{6} \frac{\beta_{2+\delta}}{y^{1+\delta}}+\frac{\beta_{2+\delta}}{y^{1+\delta}}\left(1+h y+\frac{(h y)^{2}}{2}\right)\right) \\
& =\frac{1}{A_{3}(K)}\left(1+h y+\frac{(h y)^{2}}{2}+\frac{(h y)^{3}}{6}\right) \frac{\beta_{2+\delta}}{y^{1+\delta}} \leqslant \frac{1}{A_{3}(K)} \frac{\beta_{2+\delta} e^{h y}}{y^{1+\delta}} .
\end{aligned}
$$

С другой стороны, при оценке величины $I_{2}$ было показано, что

$$
\mathbf{E} X_{1}^{*}-h=\frac{1}{f(h)}\left((1-f(h)) h+\frac{\beta_{2+\delta} e^{h y}}{y^{1+\delta}}\right) \leqslant \frac{1}{A_{3}(K)}\left(\frac{h^{2} \beta_{2+\delta}}{y^{1+\delta}}+\frac{\beta_{2+\delta} e^{h y}}{y^{1+\delta}}\right)
$$

Сравнивая эту оценку с оценкой для $h-\mathbf{E} X_{1}^{*}$, мы получаем в силу (27)

$$
\begin{aligned}
\left|\mathbf{E} X_{1}^{*}-h\right| & \leqslant \frac{1}{A_{3}(K)}\left(\frac{h^{2} \beta_{2+\delta}}{y^{1+\delta}}+\frac{\beta_{2+\delta} e^{h y}}{y^{1+\delta}}\right) \\
& \leqslant \frac{\beta_{2+\delta}}{\sqrt{n} A_{3}(K) n^{\delta / 2}}\left(\frac{(1-\gamma)^{2} x^{2}}{(x \gamma)^{1+\delta} n}+\frac{e^{\gamma(1-\gamma) x^{2}}}{(x \gamma)^{1+\delta}}\right)
\end{aligned}
$$




$$
\begin{aligned}
& \leqslant \frac{\beta_{2+\delta}}{n^{(1-\delta) / 2} A_{3}(K)}[ \frac{(1-\gamma)^{2} K^{3-\delta+4 / \delta}}{a^{2 / \delta} \gamma^{1+\delta}} \exp \left\{-\frac{4(b-c)}{\delta b^{2}} K^{2}\right\} \\
&\left.+\frac{\exp \left\{\gamma(1-\gamma) x^{2}\right\}}{\gamma^{1+\delta} x^{1+\delta}}\right] \equiv \frac{\widehat{A}_{11}(x)}{\sqrt{n}} L_{n}^{2+\delta}
\end{aligned}
$$

С помощью полученной при рассмотрении $I_{2}$ оценки $\sqrt{n}\left(h-\mathbf{E} X_{1}^{*}\right) \geqslant$ $-A_{11}(K)$, где $A_{11}(K)>0$, величина $I_{33}$ оценивается следующим образом:

$$
\begin{aligned}
I_{33} & \leqslant\left|\sqrt{n}\left(\mathbf{E} X_{1}^{*}-h\right)\right| \varphi\left(\min \left\{\gamma K-A_{11}(K), \gamma K\right\}\right) \\
& \leqslant \frac{\widehat{A}_{11}(x)}{\sqrt{2 \pi}} \exp \left\{-\frac{1}{2}\left[\gamma K-A_{11}(K)\right]^{2}\right\} L_{n}^{2+\delta} .
\end{aligned}
$$

Таким образом, получаем оценку $I_{3} \leqslant A_{16}(x) \beta_{2+\delta} / n^{\delta / 2}$, где

$$
\begin{aligned}
A_{16}(x)= & 0.3041\left(\frac{\widehat{A}_{10}(x)}{A_{7}^{3 / 2}(K)}+\frac{K^{(2+\delta)(1-\delta) / \delta}}{a^{(1-\delta) / \delta}} \exp \left\{-\frac{2(1-\delta)(b-c)}{\delta b^{2}} K^{2}\right\}\right) \\
& +\frac{\max \left\{1, A_{14}(K) \sqrt{A_{7}(K)}\right\}}{\sqrt{8 \pi}} \max \left\{A_{12}(x), \frac{\widehat{A}_{6}(x)}{A_{7}(K)}\right\} \\
& \times \exp \left\{-\frac{1}{2}\left[\max \left\{1, A_{14}(K) \sqrt{A_{7}(K)}\right\}\right]^{2}\right\} \\
& +\frac{\widehat{A}_{11}(x)}{\sqrt{2 \pi}} \exp \left\{-\frac{1}{2}\left[\gamma K-A_{11}(K)\right]^{2}\right\} .
\end{aligned}
$$

В итоге для каждого $x$ из рассматриваемого диапазона (ii) справедливо неравенство

$$
\Delta_{n}(x) \leqslant \frac{\beta_{2+\delta}}{\gamma^{2+\delta}|x|^{2+\delta} n^{\delta / 2}}+I_{1} \cdot I_{2}+2 I_{3} e^{-\left(1-\gamma^{2}\right) x^{2} / 2} \leqslant A_{17}(x) \frac{\beta_{2+\delta}}{|x|^{2+\delta} n^{\delta / 2}},
$$

где

$$
\begin{aligned}
A_{17}(x) & =A_{17}(x, \delta, a, b, c, \gamma) \\
& =\frac{1}{\gamma^{2+\delta}}+A_{1}(K) A_{2}(K) A_{15}(K)+2 A_{16}(|x|)|x|^{2+\delta} e^{-\left(1-\gamma^{2}\right) x^{2} / 2} .
\end{aligned}
$$

Покажем, что для $x \geqslant K$ при всех рассматриваемых значениях $\delta, a, b, c, \gamma$ выполняется неравенство $A_{17}(x, \delta, a, b, c, \gamma) \leqslant A_{17}(K, \delta, a, b, c, \gamma)$. Для этого, пользуясь соотношением (25), покажем, что для всех $x \geqslant K$

$$
A_{16}(x) x^{2+\delta} \exp \left\{-\frac{\left(1-\gamma^{2}\right) x^{2}}{2}\right\} \leqslant A_{16}(K) K^{2+\delta} \exp \left\{-\frac{\left(1-\gamma^{2}\right) K^{2}}{2}\right\} .
$$

Заметим прежде всего, что в силу (34) функция $x^{2+\delta} \exp \left\{-\left(1-\gamma^{2}\right) x^{2} / 2\right\}$ монотонно убывает при $x \geqslant K$. 
Далее, рассмотрим выражение $\widehat{A}_{10}(x) x^{2+\delta} \exp \left\{-\left(1-\gamma^{2}\right) x^{2} / 2\right\}$. В него входят следующие слагаемые вида $x^{r} \exp \left\{-s x^{2}\right\}$ :

$$
x^{3} \exp \left\{-\frac{(1-\gamma)^{2} x^{2}}{2}\right\} \quad \text { и } \quad\left(\widehat{A}_{8}(x)+\widehat{A}_{9}(x)\right) x^{2+\delta} \exp \left\{-\frac{\left(1-\gamma^{2}\right) x^{2}}{2}\right\} .
$$

Первое слагаемое убывает в силу (33). В выражении $\widehat{A}_{8}(x) x^{2+\delta} \exp \{-(1-$ $\left.\left.\gamma^{2}\right) x^{2} / 2\right\}$ интересующие нас слагаемые имеют вид

$$
\begin{gathered}
x^{(2+\delta)(2-\delta) / \delta} \exp \left\{-\frac{2(2-\delta)(b-c)}{\delta b^{2}} x^{2}\right\} \cdot x^{3+\delta} \exp \left\{-\frac{\left(1-\gamma^{2}\right) x^{2}}{2}\right\}, \\
\left(x^{4 / \delta+1}+x^{4 / \delta+3}\right) \exp \left\{-\frac{4(b-c)}{\delta b^{2}} x^{2}\right\} \cdot x^{2} \exp \left\{-\frac{(1-\gamma)^{2} x^{2}}{2}\right\}, \\
x^{4 / \delta+3} \exp \left\{-\left(\frac{2(2+\delta)(b-c)}{\delta b^{2}}-2 \gamma(1-\gamma)\right) x^{2}\right\} \cdot x^{2} \exp \left\{-\frac{\left(1-\gamma^{2}\right) x^{2}}{2}\right\} .
\end{gathered}
$$

Первое из них убывает, если справедливо более сильное условие (27) и условие $K^{2} \geqslant(3+\delta) /\left(1-\gamma^{2}\right)$, которое вытекает из (34). Второе слагаемое убывает, если $K^{2} \geqslant(4+3 \delta) / 8 \cdot b^{2} /(b-c)$ и $K^{2} \geqslant 2 /(1-\gamma)^{2}$. При этом оба условия слабее уже перечисленных условий (27) и (33) соответственно. Условия (26), (31) и (34) гарантируют убывание третьего слагаемого.

В выражении $\widehat{A}_{9}(x) x^{2+\delta} \exp \left\{-\left(1-\gamma^{2}\right) x^{2} / 2\right\}$ интересующий нас сомножитель имеет вид $x^{4 / \delta} \exp \left\{-2(2-\delta)(b-c) x^{2} /\left(\delta b^{2}\right)\right\} \cdot x^{4} \exp \{-(1-$ $\left.\left.\gamma^{2}\right) x^{2} / 2\right\}$, и он убывает, если выполнены условия (27) и $(34)$.

В выражениях $A_{12}(x) x^{2+\delta} \exp \left\{-\left(1-\gamma^{2}\right) x^{2} / 2\right\}$ и $\widehat{A}_{11}(x) \exp \{-(1-$ $\left.\left.\gamma^{2}\right) x^{2} / 2\right\}$ интересующие нас слагаемые имеют вид $x^{2} \exp \left\{-(1-\gamma)^{2} x^{2} / 2\right\}$ и $x \exp \left\{-(1-\gamma)^{2} x^{2} / 2\right\}$ соответственно, и они убывают в силу (33).

Рассмотрим «наихудшие» слагаемые вида $x^{r} \exp \left\{-s x^{2}\right\}$, входящие в $\widehat{A}_{6}(x) x^{2+\delta} \exp \left\{-\left(1-\gamma^{2}\right) x^{2} / 2\right\}$ :

$$
\left(x^{2-\delta}+\widehat{A}_{4}(x)+\widehat{A}_{5}(x)\right) x^{2+\delta} \exp \left\{-\frac{\left(1-\gamma^{2}\right) x^{2}}{2}\right\} .
$$

Первое слагаемое убывает в силу (34). Слагаемое вида $x^{r} \exp \left\{-s x^{2}\right\}$, присутствующее в выражении $\widehat{A}_{4}(x) x^{2+\delta} \exp \left\{-\left(1-\gamma^{2}\right) x^{2} / 2\right\}$, имеет вид $x^{4 / \delta} \exp \left\{-2(2-\delta)(b-c) x^{2} /\left(\delta b^{2}\right)\right\} \cdot x^{4} \exp \left\{-\left(1-\gamma^{2}\right) x^{2} / 2\right\}$, и оно убывает, если выполнены (27) и (34). Аналогичные рассуждения применимы к первому слагаемому вида $x^{r} \exp \left\{-s x^{2}\right\}$ в выражении $\widehat{A}_{5}(x) x^{2+\delta} \exp \{-(1-$ $\left.\left.\gamma^{2}\right) x^{2} / 2\right\}$. Второе слагаемое этого выражения $x^{4 / \delta} \exp \left\{-4(b-c) x^{2} /\left(\delta b^{2}\right)\right\}$. $x^{2} \exp \left\{-(1-\gamma)^{2} x^{2} / 2\right\}$ убывает в силу (27) и (33).

Резюмируя сказанное, мы получаем следующее утверждение.

Теорема 2. Предположим, ито $K>0, K^{2} \leqslant x^{2} \leqslant c_{n}(x ; \delta, a, b, c)$, $0<\gamma<1, a>0, b>c \geqslant 1$ mаковь, ито $A(K) \leqslant 1 / 6, A_{3}(K)>0$, 
$A_{7}(K)>0, A_{14}(K)>0$ и выполнены условия (26)-(34). Тогда для всех $n \geqslant 1$ u $F \in \mathscr{F}_{2+\delta}$

$$
|x|^{2+\delta} \Delta_{n}(x) \leqslant A_{17}(K, \delta, a, b, c, \gamma) L_{n}^{2+\delta} .
$$

3 а м е ч а н и е 2 . Несложно убедиться, что при $K \rightarrow \infty$ величины $A(K) \rightarrow 0, A_{3}(K) \rightarrow 1, A_{7}(K) \rightarrow 1, A_{14}(K) \rightarrow \infty$, так что все условия теоремы 2 , кроме (26), выполняются автоматически для любых фиксированных $a, b, c, \gamma$. Кроме того,

$$
\inf _{a, b, c, \gamma} \lim _{K \rightarrow \infty} A_{17}(K, \delta, a, b, c, \gamma)=\inf _{a, b, c, \gamma} \gamma^{-(2+\delta)}=\lim _{\gamma \rightarrow 1-} \gamma^{-(2+\delta)}=1 .
$$

При $\gamma \rightarrow 1$ справедливость условия (26) также становится очевидной для любых фиксированных $b>c \geqslant 1$.

\section{5. Основные результаты.}

Теорема 3. Для константы $U(\delta)$ в неравенстве

$$
\sup _{x \in \mathbf{R}}|x|^{2+\delta} \Delta_{n}(x) \leqslant U(\delta) L_{n}^{2+\delta}, \quad n \geqslant 1, \quad F \in \mathscr{F}_{2+\delta},
$$

справедлива оченка

$$
U(\delta)=\inf _{K, a, b, c, \gamma} \max \left\{C_{0}(\delta) K^{2+\delta}, A_{17}(K, \delta, a, b, c, \gamma), P(\delta, a, b, c, K)\right\},
$$

где инфимум берется по множеству всех значений вспомогательных параметров $a, b, c, \gamma, K$, удовлетворяющих условиям теоремь 2, а также условиям $K \geqslant 1 / \sqrt{2 \pi}$ и $a \leqslant P(\delta, a, b, c, K) / \varkappa($ величина $\varkappa=$ $0.54 \ldots$ определена в лемме 4).

Следствие 1. Неравенство (4) при всех $n \geqslant 1$ и $F \in \mathscr{F}_{2+\delta}$ справедливо с $C(\delta)=U(\delta)+C_{0}(\delta)$.

Таблица 5. Оптимальные значения $K_{0}(\delta), a_{0}(\delta), b_{0}(\delta), c_{0}(\delta), \gamma_{0}(\delta)$, доставляющие минимум в $(44)$, и соответствующие значения констант $U(\delta)$ и $C(\delta)$.

\begin{tabular}{||c|c|c|c|c|c|c|c||}
\hline$\delta$ & $K_{0}(\delta)$ & $a_{0}(\delta)$ & $b_{0}(\delta)$ & $c_{0}(\delta)$ & $\gamma_{0}(\delta)$ & $U(\delta)$ & $C(\delta)$ \\
\hline 1.0 & 3.3281 & 9.4865 & 1.5978 & 1.0144 & 0.4796 & 17.6355 & 18.1139 \\
0.9 & 3.2705 & 9.3306 & 1.5999 & 1.0209 & 0.4704 & 16.7268 & 17.2651 \\
0.8 & 3.2545 & 8.4107 & 1.6006 & 1.0211 & 0.4678 & 15.5801 & 16.1524 \\
0.7 & 3.2466 & 8.4508 & 1.6408 & 1.0466 & 0.4665 & 14.4840 & 15.0866 \\
0.6 & 3.2485 & 8.5886 & 1.6621 & 1.0699 & 0.4668 & 13.4299 & 14.0576 \\
0.5 & 3.2683 & 8.0718 & 1.6918 & 1.0865 & 0.4700 & 12.3845 & 13.0258 \\
0.4 & 3.3236 & 7.9432 & 1.7428 & 1.1165 & 0.4789 & 11.3263 & 11.9605 \\
0.3 & 3.4014 & 7.6738 & 1.7685 & 1.1339 & 0.4810 & 10.3480 & 10.9675 \\
0.2 & 3.4760 & 7.2242 & 1.7914 & 1.1466 & 0.4702 & 9.4467 & 10.0561 \\
0.1 & 3.5472 & 6.9864 & 1.8266 & 1.1665 & 0.4616 & 8.6086 & 9.2114 \\
\hline
\end{tabular}


В таблице 5 приведены значения величин $U(\delta)$ и $C(\delta)$ при некоторых $\delta \in(0,1]$. Алгоритм оптимизации в $(44)$ реализован с использованием пакета Matlab 7.10.0, при этом применялась процедура fminsearch $(.$.$) от$ аргументов $K, a, b, c, \gamma$. Оптимальные значения $K_{0}(\delta), a_{0}(\delta), b_{0}(\delta), c_{0}(\delta)$ и $\gamma_{0}(\delta)$, доставляющие минимум $U(\delta)$, также приведены в таблице 5 .

Д о к а з а т е л ь с т в о т е о р е м ы 3 . Покажем, что для любых значений параметров $K, a, b, c, \gamma$, удовлетворяющих условиям теоремы, при всех $x \in \mathbf{R}, n \geqslant 1$ и $F \in \mathscr{F}_{2+\delta}$ выполняется неравенство $|x|^{2+\delta} \Delta_{n}(x) \leqslant$ $\max \left\{C_{0}(\delta) K^{2+\delta}, A_{17}(K, \delta, a, b, c, \gamma), P(\delta, a, b, c, K)\right\} L_{n}^{2+\delta}$. Зафиксируем $K$ и $x$. Возможны два варианта:

1) $|x| \leqslant K$, тогда $|x|^{2+\delta} \Delta_{n}(x) \leqslant C_{0}(\delta) K^{2+\delta} L_{n}^{2+\delta}$;

2) $|x|>K$, тогда для заданных $a, b, c, n \geqslant 1$ и $F \in \mathscr{F}_{2+\delta}$ имеются три возможности:

2.1) $c_{n}(x ; \delta, a, b, c) \leqslant 0$, но это условие означает, что $a L_{n}^{2+\delta} \geqslant|x|^{2+\delta}$, откуда по лемме 4 получаем, что $\Delta_{n}(x) \leqslant \varkappa \leqslant P(\delta, a, b, c, K) / a \leqslant$ $P(\delta, a, b, c, K) L_{n}^{2+\delta} /|x|^{2+\delta}$

2.2) $0<c_{n}(x ; \delta, a, b, c)<x^{2}$, т.е. $x^{2} \geqslant \max \left\{(2 \pi)^{-1}, c_{n}(x ; \delta, a, b, c)\right\}$ и мы находимся в условиях теоремы 1 , согласно которой $|x|^{2+\delta} \Delta_{n}(x) \leqslant$ $P(\delta, a, b, c, x) L_{n}^{2+\delta} \leqslant P(\delta, a, b, c, K) L_{n}^{2+\delta}$, так как $|x| \geqslant K$, а функция $P(\delta, a, b, c, x)$ монотонно не возрастает по $x$;

2.3) $x^{2} \leqslant c_{n}(x ; \delta, a, b, c)$. Учитывая также условие $x^{2} \geqslant K^{2}$, по теореме 2 получаем $|x|^{2+\delta} \Delta_{n}(x) \leqslant A_{17}(K, \delta, a, b, c, \gamma) L_{n}^{2+\delta}$.

Теорема 3 доказана.

Теоремы 1, 2 и 3 позволяют указать алгоритм нахождения при каждом $0<\delta \leqslant 1$ и $x \geqslant 0$ значений функции $C(x, \delta)$, гарантирующей справедливость неравенства (6).

Теорема 4. Для любых $n \geqslant 1, F \in \mathscr{F}_{2+\delta} u K \geqslant 0$ неравенство $\sup _{|x| \geqslant K}|x|^{2+\delta} \Delta_{n}(x) \leqslant C(K, \delta) L_{n}^{2+\delta}$ справедливо с

$C(K, \delta)=\left\{\begin{array}{l}U(\delta), \quad K \leqslant K_{0}(\delta), \\ \inf _{a, b, c, \gamma} \max \left\{A_{17}(K, \delta, a, b, c, \gamma), P(\delta, a, b, c, K)\right\}, \quad K>K_{0}(\delta),\end{array}\right.$

где при каждом $K>K_{0}(\delta)$ минимум берется по множеству всех значений вспомогательных параметров $a, b, c, \gamma$, удовлетворяюших условиям теоремь 2, а также условию $a \leqslant P(\delta, a, b, c, K) / \varkappa($ величина $\varkappa=0.54 \ldots$ определена в лемме 4$), K_{0}(\delta) \geqslant 1 / \sqrt{2 \pi}$ - оптимальное значение параметра $K$ в (44).

Значения функции $C(x, \delta)$ для некоторых $\delta \in(0,1]$ и $x>K_{0}(\delta)$ приведены в таблицах 6 и 7.

Д о к аз а т е л ь с т в о т е о р е м ы 4. Очевидно, при всех $K \geqslant 0$ $\sup _{|x| \geqslant K}|x|^{2+\delta} \Delta_{n}(x) \leqslant \sup _{x \in \mathbf{R}}|x|^{2+\delta} \Delta_{n}(x) \leqslant U(\delta) L_{n}^{2+\delta}$ в силу теоремы 3.

Покажем, что при $K \geqslant K_{0}(\delta)$ для любых $n \geqslant 1, F \in \mathscr{F}_{2+\delta},|x| \geqslant K$ и $a, b, c, \gamma$, удовлетворяющих условиям теоремы, справедливо неравенство 
$|x|^{2+\delta} \Delta_{n}(x) \leqslant \max \left\{A_{17}(K, \delta, a, b, c, \gamma), P(\delta, a, b, c, K)\right\} L_{n}^{2+\delta}$. Имеются две возможности:

1) $c_{n}(K ; \delta, a, b, c) \leqslant 0$. Тогда для $|x| \geqslant K$ возможны варианты:

1.1) $c_{n}(x ; \delta, a, b, c) \leqslant 0$, но это означает, что $a L_{n}^{2+\delta} /|x|^{2+\delta} \geqslant 1$, откуда по лемме 4 получаем, что $\Delta_{n}(x) \leqslant \varkappa \leqslant P(\delta, a, b, c, K) / a \leqslant$ $P(\delta, a, b, c, K) L_{n}^{2+\delta} /|x|^{2+\delta}$;

Таблица 6. Значения функции $C(x, \delta)$ из теоремы 4 для некоторых $x$ и $\delta=1.0, \ldots, 0.6$.

\begin{tabular}{||c||c|c|c|c|c||}
\hline$x \backslash \delta$ & 1.0 & 0.9 & 0.8 & 0.7 & 0.6 \\
\hline 3.6 & 16.0383 & 14.9325 & 13.9304 & 13.0058 & 12.1428 \\
3.7 & 15.6706 & 14.5953 & 13.6128 & 12.7035 & 11.8538 \\
3.8 & 15.3061 & 14.2565 & 13.2949 & 12.4019 & 11.5664 \\
3.9 & 14.9414 & 13.9203 & 12.9793 & 12.1043 & 11.2847 \\
4.0 & 14.5837 & 13.5890 & 12.6694 & 11.8129 & 11.0099 \\
5.0 & 11.4967 & 10.7357 & 10.0227 & 9.3529 & 8.7234 \\
6.0 & 9.1885 & 8.6148 & 8.0740 & 7.5641 & 7.0839 \\
7.0 & 7.4723 & 7.0385 & 6.6280 & 6.2396 & 5.8722 \\
8.0 & 6.2210 & 5.8870 & 5.5697 & 5.2682 & 4.9819 \\
9.0 & 5.3089 & 5.0452 & 4.7938 & 4.5540 & 4.3254 \\
10.0 & 4.6334 & 4.4199 & 4.2156 & 4.0202 & 3.8332 \\
15.0 & 2.9524 & 2.8536 & 2.7580 & 2.6653 & 2.5755 \\
20.0 & 2.3099 & 2.2494 & 2.1903 & 2.1327 & 2.0764 \\
50.0 & 1.4388 & 1.4220 & 1.4054 & 1.3890 & 1.3727 \\
100 & 1.1565 & 1.1510 & 1.1456 & 1.1402 & 1.1349 \\
200 & 1.0605 & 1.0585 & 1.0566 & 1.0546 & 1.0527 \\
500 & 1.0252 & 1.0243 & 1.0235 & 1.0227 & 1.0219 \\
1000 & 1.0131 & 1.0126 & 1.0122 & 1.0118 & 1.0114 \\
$\infty$ & $1+$ & $1+$ & $1+$ & $1+$ & $1+$ \\
\hline
\end{tabular}

1.2) $0<c_{n}(x ; \delta, a, b, c) \leqslant x^{2}$. Поскольку при этом также $x^{2} \geqslant K^{2} \geqslant$ $(2 \pi)^{-1}$, по теореме 1 заключаем, что $|x|^{2+\delta} \Delta_{n}(x) \leqslant P(\delta, a, b, c, x) L_{n}^{2+\delta} \leqslant$ $P(\delta, a, b, c, K) L_{n}^{2+\delta}$, т.к. $|x| \geqslant K$, а функция $P(\delta, a, b, c, x)$ монотонно не возрастает по $x$;

1.3) $c_{n}(x ; \delta, a, b, c) \geqslant x^{2} \geqslant K^{2}$. Для этого случая по теореме 2 получаем $|x|^{2+\delta} \Delta_{n}(x) \leqslant A_{17}(K, \delta, a, b, c, \gamma) L_{n}^{2+\delta}$.

2) $c_{n}(K ; \delta, a, b, c)>0$. Отсюда вытекает, что $c_{n}(x ; \delta, a, b, c)>$ $c_{n}(K ; \delta, a, b, c)>0$ для всех $|x| \geqslant K$, следовательно для $c_{n}(x ; \delta, a, b, c)$ возможны два варианта:

2.1) $0<c_{n}(x ; \delta, a, b, c) \leqslant x^{2}$. Поскольку при этом также $x^{2} \geqslant K^{2} \geqslant$ $(2 \pi)^{-1}$, по теореме 1 заключаем, что $|x|^{2+\delta} \Delta_{n}(x) \leqslant P(\delta, a, b, c, x) L_{n}^{2+\delta} \leqslant$ $P(\delta, a, b, c, K) L_{n}^{2+\delta}$; 
Таблица 7. Значения функции $C(x, \delta)$ из теоремы 4 для некоторых $x$ и $\delta=0.5, \ldots, 0.1$.

\begin{tabular}{||c||c|c|c|c|c||}
\hline$x \backslash \delta$ & 0.5 & 0.4 & 0.3 & 0.2 & 0.1 \\
\hline 3.6 & 11.3303 & 10.5615 & 9.8322 & 9.1409 & 8.4863 \\
3.7 & 11.0530 & 10.2962 & 9.5797 & 8.9017 & 8.2609 \\
3.8 & 10.7798 & 10.0368 & 9.3343 & 8.6706 & 8.0441 \\
3.9 & 10.5133 & 9.7848 & 9.0974 & 8.4482 & 7.8366 \\
4.0 & 10.2539 & 9.5411 & 8.8687 & 8.2351 & 7.6384 \\
5.0 & 8.1311 & 7.5747 & 7.0521 & 6.5612 & 6.1005 \\
6.0 & 6.6315 & 6.2046 & 5.8036 & 5.4253 & 5.0699 \\
7.0 & 5.5247 & 5.1961 & 4.8854 & 4.5918 & 4.3143 \\
8.0 & 4.7101 & 4.4519 & 4.2068 & 3.9741 & 3.7532 \\
9.0 & 4.1074 & 3.8997 & 3.7016 & 3.5129 & 3.3330 \\
10.0 & 3.6542 & 3.4831 & 3.3194 & 3.1628 & 3.0130 \\
15.0 & 2.4885 & 2.4043 & 2.3227 & 2.2437 & 2.1671 \\
20.0 & 2.0216 & 1.9681 & 1.9159 & 1.8649 & 1.8152 \\
50.0 & 1.3566 & 1.3407 & 1.3249 & 1.3093 & 1.2939 \\
100 & 1.1296 & 1.1242 & 1.1189 & 1.1136 & 1.1083 \\
200 & 1.0508 & 1.0488 & 1.0469 & 1.0449 & 1.0430 \\
500 & 1.0211 & 1.0203 & 1.0195 & 1.0187 & 1.0179 \\
1000 & 1.0109 & 1.0105 & 1.0101 & 1.0097 & 1.0093 \\
$\infty$ & $1+$ & $1+$ & $1+$ & $1+$ & $1+$ \\
\hline
\end{tabular}

2.2) $c_{n}(x ; \delta, a, b, c) \geqslant x^{2} \geqslant K^{2}$, и по теореме 2 получаем $|x|^{2+\delta} \Delta_{n}(x) \leqslant$ $A_{17}(K, \delta, a, b, c, \gamma) L_{n}^{2+\delta}$. Теорема доказана.

Из теоремы 4 можно получить асимптотические при $x \rightarrow \infty$ оценки функций $C_{*}(x, \delta)$ и $C^{*}(x, \delta)$.

Следствие 2. Для функций

$$
\begin{aligned}
& C_{*}(x, \delta)=\limsup _{\ell \rightarrow 0} \sup _{n \geqslant 1,} \sup _{F \in \mathscr{F}_{2+\delta}: L_{n}^{2+\delta}=\ell}|t|^{2+\delta} \Delta_{n}(t) / \ell, \quad x \geqslant 0, \\
& C^{*}(x, \delta)=\sup _{n \geqslant 1, \mathscr{F}_{F \in \mathscr{F}_{2+\delta}}} \sup _{|t| \geqslant x}|t|^{2+\delta} \Delta_{n}(t) / L_{n}^{2+\delta}, \quad x \geqslant 0
\end{aligned}
$$

при всех $0<\delta \leqslant 1$ справедливь асимптотические оченки $\lim _{x \rightarrow \infty} C_{*}(x, \delta) \leqslant \lim _{x \rightarrow \infty} C^{*}(x, \delta) \leqslant 1$, при этом для $\delta=1$ в обоих случаях имеет место знак равенства.

Д о к а з а т е л ь с т в о. Неравенство $C_{*}(x, \delta) \leqslant C^{*}(x, \delta)$ очевидно при всех $x \geqslant 0$ и $0<\delta \leqslant 1$. Из теоремы 4 для всех $0<a \leqslant 1 / \varkappa, b>c \geqslant 1$ и $0<\gamma<1$ таких, что $2(b-c) / b^{2}>\gamma(1-\gamma)$, получаем оценку

$$
\begin{aligned}
\lim _{x \rightarrow \infty} C^{*}(x, \delta) & \leqslant \lim _{x \rightarrow \infty} C(x, \delta) \leqslant \lim _{x \rightarrow \infty}\left(A_{17}(x, \delta, a, b, c, \gamma) \vee P(\delta, a, b, c, x)\right) \\
& \leqslant \gamma^{-(2+\delta)} \vee b^{2+\delta}
\end{aligned}
$$


(знаком $\vee$ обозначен максимум). Выбирая теперь, например, $b=1 / \gamma$, $c=2 /(1+\gamma)$ (можно убедиться, что такой выбор параметров $b$ и $c$ является допустимым при любых значениях $0<\gamma<1$ ) и устремляя $\gamma \rightarrow 1-$, окончательно получаем оценку $\lim _{x \rightarrow \infty} C^{*}(x, \delta) \leqslant 1$. При этом для случая $\delta=1$ в последнем неравенстве имеет место знак равенства, поскольку из результата работы Чистякова [27] вытекает нижняя оценка $\lim _{x \rightarrow \infty} C_{*}(x, 1) \geqslant 1$. Следствие доказано.

В заключение авторы выражают глубокую признательность В. Ю. Королеву за стимулирующие дискуссии и постоянное внимание к работе, Ю. В. Прохорову за поддержку, а также Л. Падитцу за неоценимую библиографическую помощь.

\section{СПИСОК ЛИТЕРАТУРЫ}

1. Бикялис А. Оценки остаточного члена в центральной предельной теореме. Литов. матем. сб., 1966, т. 6, в. 3, с. 323-346.

2. Бикялис $A$. О точности аппроксимации распределений сумм независимых одинаково распределенных случайных величин нормальным распредедением. - Литов. матем. сб., 1971, т. 11, в. 2, с. 237-240.

3. Бхаттачария Р. Н., Ранга Рао Р. Аппроксимация нормальным распределением. М.: Наука, 1982, 286 с.

4. Гавриленко C. В. Уточнение неравномерных оценок скорости сходимости распределений пуассоновских случайных сумм к нормальному закону. - Информатика и ее применения, 2011 , т. 5 , в. 1 , с. $12-24$.

5. Григорьева М. Е., Шевцова И.Г. Уточнение неравенства Каца-Берри-Эссеена. - Информатика и ее применения, 2010, т. 4, в. 2, с. 78-85.

6. Золотарев В. М. Современная теория суммирования независимых случайных величин. М.: Наука, 1986, 415 с.

7. Колмогоров A. Н. Некоторые работы последних лет в области предельных теорем теории вероятностей. - Вестник Моск. ун-та, сер. физ.-матем. и естеств. наук, 1953, в. 7, № 10, с. 29-38.

8. Королев В.Ю., Бенинг В.Е., Шоргин С.Я. Математические основы теории риска. М.: Физматлит, 2007.

9. Королев В. Ю., Попов С. В. Уточнение оценок скорости сходимости в центральной предельной теореме при отсутствии моментов порядков, бо́льших второго. Теория вероятн. и ее примен., 2011, т. 56, в. 4, с. 797-805.

10. Королев В. Ю., Шевцова И. Г. Уточнение неравенства Берри-Эссеена. - Докл. PAH, 2010, т. 430 , в. 6 , с. $738-742$.

11. Королев В.Ю., Шевцова И.Г. О верхней оценке абсолютной постоянной в неравенстве Берри-Эссеена. - Теория вероятн. и ее примен., 2009, т. 54, в. 4, c. $671-695$.

12. Королев В. Ю., Шевцова И. Г. Уточнение неравенства Берри-Эссеена с приложениями к пуассоновским и смешанным пуассоновским случайным суммам. Обозрение прикл. и промышл. матем., 2010, т. 17, в. 1, с. 25-56.

13. Мешалкин Л. Д., Рогозин Б. А. Оценка расстояния между функциями распределения по близости их характеристических функций и ее применение к центральной предельной теореме. - В сб. «Предельные теоремы теории вероятностей». Ташкент: изд-во АН УзССР, 1963, с. 49-55.

14. Мирахмедов Ш. А. Об абсолютной постоянной в неравномерной оценке скорости сходимости в центральной предельной теореме. - Изв. АН УзССР, сер. физ.-мат. наук, 1984, в. 4, с. 26-30. 
15. Нагаев C. В. Некоторые предельные теоремы для больших уклонений. - Теория вероятностей и ее применения, 1965, т. 10, в. 2, с. 231-254.

16. Нефедова Ю. С., Шевцова И. Г. О точности нормальной аппроксимации для распределений пуассоновских случайных сумм. - Информатика и ее применения, 2011 , т. 5, в. 1, с. $39-45$.

17. Никулин В. Н. Неравномерные оценки остаточного члена в центральной предельной теореме. - Теория вероятн. и ее примен., 1992, т. 34, в. 4, с. 831-832.

18. Осипов Л. В. Уточнение теоремы Линдеберга. - Теория вероятн. и ее примен., 1966, т. 11 , в. 2 , с. $339-342$.

19. Осипов Л. В., Петров В. В. Об оценке остаточного члена в центральной предельной теореме. - Теория вероятн. и ее примен., 1967, т. 12, в. 2, с. 322-329.

20. Падити Л., Мирахмедов Ш. А. Письмо в редакцию (Замечание к оценке абсолютной постоянной в неравномерной оценке скорости сходимости в ц.п.т.) - Изв. АН УзССР, сер. физ.-мат. наук, 1986, в. 3, с. 80.

21. Петров В.В. Одна оценка отклонения распределения суммы независимых случайных величин от нормального закона. - Докл. АН СССР, 1965, т. 160, в. 5, c. $1013-1015$.

22. Петров В. В. Суммы независимых случайных величин. М.: Наука, 1972, 416 с.

23. Петров B. В. Предельные теоремы для сумм независимых случайных величин. М.: Наука, 1987, 320 с.

24. Петров В. В. Об оценке остаточного члена в центральной предельной теореме. Записки научных семинаров ПОМИ, 2007, т. 341, с. 142-146.

25. Розовский Л. В. Неравномерная оценка остаточного члена в центральной предельной теореме. - Записки научных семинаров ПОМИ, 2007, т. 351, с. 238-241.

26. Тюрин И. С. Уточнение верхних оценок констант в теореме Ляпунова. - Успехи матем. наук, 2010 , т. 65 , в. 3, с. 201-202.

27. Чистяков Г. П. Об одной задаче А.Н. Колмогорова. - Записки научных семинаров ЛОМИ, 1990, т. 184, с. 289-319.

28. Шевцова И.Г. О точности нормальной аппроксимации для распределений пуассоновских случайных сумм. - Обозрение прикл. и промышл. матем., 2007, т. 14, в. 1, c. $3-28$.

29. Шевцова И. Г. Об асимптотически правильных постоянных в неравенстве БерриЭссеена-Каца. - Теория вероятностей и ее применения, 2010, т. 55, в. 2, с. 271304.

30. Bening V., Korolev V. Generalized Poisson Models and their Applications in Insurance and Finance. Utrecht: VSP, 2002.

31. Berry A. C. The accuracy of the Gaussian approximation to the sum of independent variates. - Trans. Amer. Math. Soc., 1941, v. 49, p. 122-136.

32. Chen L. H. Y., Shao Q. M. A non-uniform Berry-Esseen bound via Steins method. Probability Theory and Related Fields, 2001, v. 120, p. 236-254

33. Cramér H. Sur un nouveau théorèm-limite de la théorie des probabilités. - Actualités scientifiques et industrielles, 1938, № 736, Paris.

34. Esseen C.-G. On the Liapounoff limit of error in the theory of probability. - Ark. Mat. Astron. Fys., 1942, v. A28, № 9, p. 1-19.

35. Esseen C.-G. A moment inequality with an application to the central limit theorem. Skand. Aktuarietidskr., 1956, v. 39, p. 160-170.

36. Esseen C.-G. Fourier analysis of distribution functions: a mathematical study of the Laplace-Gaussian law. - Acta Math., 1945, v. 77, p. 1-125.

37. Hoeffding $W$. The extrema of the expected value of a function of independent random variables. - Ann. Math. Statist., 1948, v. 19, p. 239-325.

38. Heyde C. C. A nonuniform bound on convergence to normality. - Ann. Probab., 1975, v. 3, № 5, p. 903-907.

39. Katz M. L. Note on the Berry-Esseen theorem. - Ann. Math. Statist., 1963, v. 34, p. $1107-1108$.

40. Korolev V., Shevtsova I. An improvement of the Berry-Esseen inequality with applications to Poisson and mixed Poisson random sums. - Scandinavian Actuarial Journal, 2010, Online first: http://www.informaworld.com/10.1080/03461238.2010.485370, 04 June 2010. 
41. Королев В.Ю., Попов С. В. Уточнение оценок скорости сходимости в центральной предельной теореме при ослабленных моментных условиях. - Докл. РАН, 2012 (в печати).

42. Michel $R$. On the accuracy of nonuniform Gaussian approximation to the distribution functions of sums of independent and identically distributed random variables. - Z . Wahrsch. verw. Geb., 1976, v. 35, № 4, p. 337-347.

43. Michel $R$. On the constant in the nonuniform version of the Berry-Esseen theorem. Z. Wahrsch. verw. Geb., 1981, v. 55, p. 109-117.

44. Michel R. On Berry-Esseen results for the compound Poisson distribution. - Insurance: Mathematics and Economics, 1993, v. 13, № 1, p. 35-37.

45. Nakata T. A nonuniform bound on convergence to normality for independent random variables. - Advances in Applied Probability, 1977, v. 11, № 2, p. 285-286

46. Neammanee $K$. On the constant in the nonuniform version of the Berry-Esseen theorem. - International Journal of Mathematics and Mathematical Sciences, 2005, v. 12, p. $1951-1967$.

47. Neammanee K., Thongtha $P$. Improvement of the non-uniform version of Berry-Esseen inequality via Paditz-Siganov theorems. - Journal of Inequalities in Pure and Applied Mathematics, 2007, v. 8, № 4, p. 92.

48. Nikulin $V$. An algorithm to estimate a nonuniform convergence bound in the central limit theorem, 2010, http://arxiv.org/PS_cache/arxiv/pdf/1004/1004.0552v1.pdf

49. Nikulin V., Paditz L. A note on nonuniform CLT-bounds. - 7th Vilnius Conference on Probability Theory and 22nd European Meeting of Statisticians. Abstracts. 1998, p. 358-359.

50. Paditz L. Abschätzungen der Konvergenzgeschwindigkeit im zentralen Grenzwertsatz. — Wiss. Z. der TU Dresden, 1976, v. 25, p. 1169-1177.

51. Paditz L. Über die Annäherung der Verteilungsfunktionen von Summen unabhängiger Zufallsgrößen gegen unbegrenzt teilbare Verteilungsfunktionen unter besonderer beachtung der Verteilungsfunktion der standardisierten Normalverteilung. — Dissertation A. Technische Universität Dresden. Dresden, 1977.

52. Paditz L. Abschätzungen der Konvergenzgeschwindigkeit zur Normalverteilung unter Voraussetzung einseitiger Momente. - Math. Nachr., 1978, v. 82, p. 131-156.

53. Paditz L. Über eine Fehlerabschätzung im zentralen Grenzwertsatz. — Wiss. Z. der TU Dresden, 1979, v. 28, № 5, p. 1197-1200.

54. Paditz L. Bemerkungen zu einer Fehlerabschätzung im zentralen Grenzwertsatz. In: Wiss. Z. Hochschule für Verkehrswesen «Friedrich List», 1980, v. 27, № 4, p. 829 837.

55. Paditz L. Einseitige Fehlerabschätzungen im zentralen Grenzwertsatz. - Math. Operationsforsch. und Statist., ser. Statist., 1981, v. 12, p. 587-604.

56. Paditz L. On error-estimates in the central limit theorem for generalized linear discounting. - In: Math. Operationsforsch. u. Statist., Ser. Statistics, 1984, v. 15, № 4, p. $601-610$.

57. Paditz L. Über eine Fehlerabschätzung im zentralen Grenzwertsatz. - Wiss. Z. Hochschule für Verkehrswesen «Friedrich List». Dresden. 1986, v. 33, № 2, p. 399-404.

58. Paditz L. On the analytical structure of the constant in the nonuniform version of the Esseen inequality. — Statistics (Berlin: Akademie-Verlag), 1989, v. 20, № 3, p. 453464.

59. Rychlik Z. Nonuniform central limit bounds and their applications. - Теория вероятн. и ее примен., 1983, т. 28, в. 3, с. 646-652.

60. Thongtha P., Neammanee $K$. Refinement on the constants in the non-uniform version of the Berry-Esseen theorem. - Thai Journal of Mathematics, 2007, v. 5, p. 1-13.

61. Tysiak W. Gleichmäßige und nicht-gleichmäßige Berry-Esseen-Abschätzungen. Dissertation. Wuppertal, 1983. 